\title{
Diffusion coefficient matrix of the strongly interacting quark-gluon plasma
}

\author{
Jan A. Fotakis $\odot,{ }^{1, *}$ Olga Soloveva $\odot,{ }^{1}$ Carsten Greiner, ${ }^{1}$ Olaf Kaczmarek $\odot,{ }^{2,3}$ and Elena Bratkovskaya ${ }^{4,1}$ \\ ${ }^{1}$ Institut für Theoretische Physik, Johann Wolfgang Goethe-Universität, \\ Max-von-Laue-Strasse 1, D-60438 Frankfurt am Main, Germany \\ ${ }^{2}$ Fakultät für Physik, Universität Bielefeld, D-33615 Bielefeld, Germany \\ ${ }^{3}$ Key Laboratory of Quark and Lepton Physics (MOE) and Institute of Particle Physics, \\ Central China Normal University, Wuhan 430079, China \\ ${ }^{4}$ GSI Helmholtzzentrum für Schwerionenforschung GmbH, Planckstrasse 1, \\ D-64291 Darmstadt, Germany
}

(Received 23 February 2021; accepted 13 July 2021; published 16 August 2021)

\begin{abstract}
We study the diffusion properties of the strongly interacting quark-gluon plasma (sQGP) and evaluate the diffusion coefficient matrix for the baryon (B), strange (S) and electric (Q) charges- $\kappa_{q q^{\prime}}$ $\left(q, q^{\prime}=\mathrm{B}, \mathrm{S}, \mathrm{Q}\right)$ and show their dependence on temperature $T$ and baryon chemical potential $\mu_{\mathrm{B}}$. The nonperturbative nature of the sQGP is evaluated within the dynamical quasiparticle model (DQPM) which is matched to reproduce the equation of state of the partonic matter above the deconfinement temperature $T_{c}$ from lattice QCD. The calculation of diffusion coefficients is based on two methods: (i) the ChapmanEnskog method for the linearized Boltzmann equation, which allows to explore nonequilibrium corrections for the phase-space distribution function in leading order of the Knudsen numbers as well as (ii) the relaxation time approximation (RTA). In this work we explore the differences between the two methods. We find a good agreement with the available lattice QCD data in case of the electric charge diffusion coefficient (or electric conductivity) at vanishing baryon chemical potential as well as a qualitative agreement with the recent predictions from the holographic approach for all diagonal components of the diffusion coefficient matrix. The knowledge of the diffusion coefficient matrix is also of special interest for more accurate hydrodynamic simulations.
\end{abstract}

DOI: 10.1103/PhysRevD.104.034014

\section{INTRODUCTION}

An exploration of the properties of hot and dense matter-created in heavy-ion collisions (HICs) at relativistic energies-is the focus of extensive research. It is the primary goal of experimental programs of the LHC (LargeHadron-Collider) at CERN, the RHIC (Relativistic Heavy Ion Collider) at BNL, the future FAIR (Facility for Antiproton and Ion Research) at GSI, and the NICA (Nuclotron-based Ion Collider fAcility) facility at JINR, which reproduce in the laboratory the extreme conditions of the early stages of our universe by "tiny bangs." In the central region of heavy-ion collisions the deconfined QCD (quantum chromodynamics) matter-a quark-gluon plasma (QGP) - is created which can achieve an approximate local equilibrium and exhibit hydrodynamic flow [1-3]. The

fotakis@itp.uni-frankfurt.de

Published by the American Physical Society under the terms of the Creative Commons Attribution 4.0 International license. Further distribution of this work must maintain attribution to the author(s) and the published article's title, journal citation, and DOI. Funded by SCOAP . hydrodynamic behavior of the fluid can be characterized by transport coefficients such as shear $\eta$, bulk $\zeta$ viscosities, and diffusion coefficients $\kappa$, which describe the fluids dissipative corrections at leading order. The interpretation of the experimental data, and especially the elliptic flow $v_{2}$, in terms of the hydrodynamic models showed that the QGP behaves almost as a nearly perfect fluid with a very low shear viscosity to entropy density $(s)$ ratio, $\eta / s$, which reflects that its properties correspond to nonperturbative, strongly interacting matter [4-6].

By performing an experimental energy scan of HICs one can explore the different stages of the QCD phase diagram. At ultrarelativistic heavy-ion collisions at LHC and RHIC energies, the QGP is created at very large temperatures $T$ and almost zero or low baryon chemical potential $\mu_{\mathrm{B}}$, where according to lattice QCD (1QCD) results [7,8] the transition from the QGP to the hadronic matter is a crossover. By reducing the collision energy one can also explore the large $\mu_{\mathrm{B}}$ region where one might expect the existence of a critical point and a 1st order phase transition. Such conditions are presently under investigation within the RHIC BES (Beam Energy Scan) experiments and in future by the FAIR and NICA facilities. 
The theoretical description of the QCD matter at finite $\mu_{\mathrm{B}}$, and especially in the vicinity of the critical point, requires an appropriate description of transport of conserved chargesbaryon $B$, strangeness $S$, and electric $Q$ charges. In order to study the phenomenon of baryon-stopping, the baryon diffusion was recently introduced to various fluid dynamic models [9-11]. Moreover, the baryon diffusion coefficient has been studied in Refs. [12-18].

In the recent past we have addressed the coupling of the conserved baryon number, strangeness and electric charge; the diffusion coefficient matrix $\left(\kappa_{q q^{\prime}}\right.$, where $\left.q, q^{\prime}=\mathrm{B}, \mathrm{S}, \mathrm{Q}\right)$ was introduced and evaluated for a hadron gas and a simple model for quark-gluon plasma (QGP) [15,17]. These investigations were followed by a more extended study in the hadronic phase from kinetic theory in the case of the electric cross-conductivities [19]. Furthermore, a first study on the impact of the coupling of baryon number and strangeness was provided in Ref. [17]. For this the diffusion coefficient matrix of hot and dense nuclear matter has to be investigated thoroughly being important for accurate hydrodynamic simulations. It was further motivated that the off-diagonal coefficients may have implications on the chemical-composition of the hadronic phase [19].

The study of the transport of conserved electric charge $Q$ during heavy-ion collisions has been in the focus of intensive research. Due to its importance for the description of soft photon spectra and rates [20-23] as well as for hydrodynamic approaches modeling the generation and evolution of electromagnetic fields [24-27], much attention was paid to the electric conductivity within different theoretical approaches for the evaluation of the properties of the partonic and hadronic matter [13-15,17-19,28-42].

The exploration of the QGP properties at finite $\left(T, \mu_{\mathrm{B}}\right)$ are of special interest for an understanding of the phase transition. The transport properties of the strongly interacting QGP has been studied using the dynamical quasi-particle model (DQPM) [43-47] that is matched to reproduce the equation of state of the partonic system above the deconfinement temperature $T_{c}$ from lattice QCD. The DQPM is based on a propagator representation with complex self energies which describes the degrees of freedom of the QGP in terms of strongly interacting dynamical quasiparticles which reflect the nonperturbative nature of the QCD in the vicinity of the phase transition where the QCD coupling grows rapidly with decreasing temperature according to IQCD calculations [48]. Moreover, the DQPM allows to explore the properties of the QGP at finite $\left(T, \mu_{\mathrm{B}}\right)$, expressed in terms of transport coefficients such as shear $\eta$, bulk $\zeta$ viscosities, baryon diffusion coefficients $\kappa_{B}$ and electric conductivity $\sigma_{0}$ based on the RTA (relaxation time approximation) $[18,47,49]$.

We note that an important advantage of a propagator based approach is that one can formulate a consistent thermodynamics [50] and a causal theory for nonequilibrium dynamics on the basis of Kadanoff-Baym equations [51]. This allows us to use the DQPM for the description of the partonic interactions and parton properties in the microscopic parton-hadron-string dynamics (PHSD) transport approach [46,52-55] and to study the QGP properties out-of equilibrium as created in HICs as well as in equilibrium by performing box calculations with periodic boundary conditions [56]. Moreover, the $\left(T, \mu_{\mathrm{B}}\right)$ dependence of partonic properties and interaction cross sections have been explored in a more recent study within PHSD 5.0 [49,57,58].

We note that the studies of transport coefficients $\left(\eta / s, \zeta / s, \kappa_{B}, \sigma_{0}\right)$ within the DQPM (and PHSD) has been based on the relaxation-time approximation (RTA) as incorporated in Refs. [59-62] as well as on the Kubo formalism [63-66] for $\eta / s$ (cf. [49,56]). In Refs. [15,17,40] the evaluation of the diffusion coefficient matrix has been done within the Chapman-Enskog method [67] which allows us to explore nonequilibrium corrections for the phase-space distribution function in leading order of the Knudsen numbers.

In the present study we combine the developments of Refs. [15,17,18] and evaluate the diffusion coefficient matrix of the strongly interacting nonperturbative QGP at finite $\left(T, \mu_{\mathrm{B}}\right)$, with properties described by the DQPM model, based on recently explored the ChapmanEnskog method [15,17,40]. This allows us to explore the influence of traces of nonequilibrium effects by accounting for the higher modes of the distribution function on the transport properties and compare the results with the often used kinetic RTA approximation. We provide the $\left(T, \mu_{\mathrm{B}}\right)$ dependence of the diffusion coefficients $\kappa_{q q^{\prime}}$ for $q, q^{\prime}=\mathrm{B}, \mathrm{S}, \mathrm{Q}$ charges for baryon chemical potentials $\mu_{\mathrm{B}} \leq 0.5 \mathrm{GeV}$, where the phase transition is a rapid crossover.

This paper is structured as follows. In Sec. II we provide a short review of the basic definitions and conventions, followed by a reminder of the basic ideas of the ChapmanEnskog method and its relaxation time approximation, which was used to evaluate the diffusion coefficient matrix in Refs. $[15,17,40]$, and a short review of the dynamical quasi-particle model (DQPM) [18,43-45,53] in Sec. II C. In the preface of Sec. III we explain how to achieve results for the diffusion matrix from the DQPM by using the Chapman-Enskog method, and we demonstrate the differences between various assumptions in Sec. III A by providing an simple example. Finally, we provide and discuss improved results for all diffusion coefficients and conductivities and compare them to the available results from other approaches.

\section{FOUNDATIONS}

Let $x \equiv x^{\mu}$ be the four-coordinate and $k \equiv k^{\mu}$ the fourmomentum. The single-particle distribution function, $f_{i, \mathbf{k}} \equiv f_{i}(x, k)$, of a multi-component quasiparticle system obeys the effective Boltzmann equation [68] 


$$
k_{i}^{\mu} \partial_{\mu} f_{i, \mathbf{k}}+\frac{1}{2} \partial_{\mu}\left(m_{i}^{2}\right) \frac{\partial}{\partial k_{i, \mu}} f_{i, \mathbf{k}}=\sum_{j=1}^{N_{\text {species }}} C_{i j}(x, k),
$$

where $C_{i j}$ is the collision term and the masses depend on temperature and chemical potentials, i.e., $m_{i} \equiv m_{i}\left(T, \mu_{\mathrm{B}}, \mu_{\mathrm{Q}}, \mu_{\mathrm{S}}\right)$. The (local) equilibrium state of the system is described by

$$
f_{i, \mathbf{k}}^{(0)}=\frac{g_{i}}{\exp \left(u_{\mu} k_{i}^{\mu} / T-\mu_{i} / T\right)-a_{i}}
$$

where $\mu_{i}=B_{i} \mu_{\mathrm{B}}+Q_{i} \mu_{\mathrm{Q}}+S_{i} \mu_{\mathrm{S}}$ is the chemical potential, $g_{i}$ is the degeneracy of the $i$ th species and

$$
a_{i} \equiv \begin{cases}+1 & \text { (Bosons }) \\ -1 & (\text { Fermions }) \\ 0 & \text { (Classical })\end{cases}
$$

Further, we define in short hand notation:

$\tilde{f}_{i, \mathbf{k}}^{(0)} \equiv 1+a_{i} \frac{f_{i, \mathbf{k}}^{(0)}}{g_{i}}=1+\frac{a_{i}}{\exp \left(u_{\mu} k_{i}^{\mu} / T-\mu_{i} / T\right)-a_{i}}$.

Furthermore, the isotropic local equilibrium pressure is determined by the temperature and chemical potentials, $P_{0} \equiv P_{0}\left(T, \mu_{\mathrm{B}}, \mu_{\mathrm{Q}}, \mu_{\mathrm{S}}\right)$. In this work, we adapt the isotropic pressure from lattice QCD [69,70]. From the equation of state the energy density and the net charge densities are defined:

$\epsilon \equiv \epsilon\left(T, \mu_{\mathrm{B}}, \mu_{\mathrm{Q}}, \mu_{\mathrm{S}}\right), \quad n_{q} \equiv n_{q}\left(T, \mu_{\mathrm{B}}, \mu_{\mathrm{Q}}, \mu_{\mathrm{S}}\right)$,

$q \in\{\mathrm{B}, \mathrm{Q}, \mathrm{S}\}$.

In kinetic theory the net charge densities are defined as:

$$
n_{q}=\sum_{i=1}^{N_{\text {species }}} q_{i} \int \mathrm{d} K_{i} E_{i, \mathbf{k}} f_{i, \mathbf{k}}^{(0)}, \quad q \in\{\mathrm{B}, \mathrm{Q}, \mathrm{S}\}
$$

where $q$ is the type of the conserved quantum number, i.e., namely baryon number $\mathrm{B}$, strangeness $\mathrm{S}$ or electric charge Q, and $q_{i}$ is the quantum number (of type $q$ ) of the $i$ th species. In this work we assume a partonic system with three flavors and thus the following particle species: up$(u)$, down- $(d)$, strange-quark $(s)$, the gluon $(g)$, and the corresponding anti-particles. Furthermore, the Landau matching conditions were assumed [71]:

$$
\sum_{i=1}^{N_{\text {species }}} q_{i} \int \mathrm{d} K_{i} E_{i, \mathbf{k}}\left(f_{i, \mathbf{k}}-f_{i, \mathbf{k}}^{(0)}\right)=0
$$

using the notation

$$
\mathrm{d} K_{i} \equiv \frac{\mathrm{d}^{3} \mathbf{k}_{i}}{(2 \pi)^{3} E_{i, \mathbf{k}}}
$$

with the on-shell energy $E_{i, \mathbf{k}}=\sqrt{m_{i}^{2}+\mathbf{k}_{i}^{2}}$.

An (unpolarized) interaction is characterized by the invariant matrix-element $\overline{\mathcal{M}}_{i_{1} \ldots i_{n} \rightarrow j_{1} \ldots j_{m}} \equiv \overline{\mathcal{M}}\left(k_{i_{1}} \ldots k_{i_{n}} \rightarrow\right.$ $\left.p_{j_{1}} \ldots p_{j_{m}}\right)$, which is averaged over the ingoing spin-states and is summed over the outgoing spin-states. The differential cross section for a binary process of on-shell particles $(i+j \rightarrow a+b)$ in the center-of-momentum frame (CM), where the momenta of the colliding particles obey $\mathbf{k}_{i}+\mathbf{k}^{\prime}{ }_{j}=\mathbf{p}_{a}+\mathbf{p}^{\prime}{ }_{b}=\mathbf{P}=0$ and $k_{i}^{0}+k_{j}^{\prime 0}=\sqrt{s}=p_{i}^{0}+p_{j}^{\prime 0}$, is given by

$$
\mathrm{d} \sigma_{i j \rightarrow a b}(\sqrt{s}, \Omega)=\frac{1}{64 \pi^{2} s} \frac{p_{\text {out }}}{p_{\text {in }}}|\overline{\mathcal{M}}|^{2} \mathrm{~d} \Omega,
$$

where $s$ in the Mandelstam variable and $\mathrm{d} \Omega$ is the differential solid angle corresponding to one of the final particles. The momenta of the initial $\left(p_{\text {in }}\right)$ and final particles $\left(p_{\text {out }}\right)$ in the $\mathrm{CM}$ frame are found to be

$$
p_{i}=\frac{\sqrt{\left(s-\left(m_{i}+m_{i}^{\prime}\right)^{2}\right)\left(s-\left(m_{i}-m_{i}^{\prime}\right)^{2}\right)}}{2 \sqrt{s}},
$$

where $i=$ in/out, $m_{i}$ and $m_{i}^{\prime}$ being the masses of the colliding partons. The total cross section is obtained via:

$$
\begin{aligned}
\sigma_{\text {tot }}^{i j \rightarrow a b}(\sqrt{s}) & \equiv 2 \pi \gamma_{i j} \int \mathrm{d} \cos (\vartheta) \frac{\mathrm{d}}{\mathrm{d} \Omega} \sigma_{i j \rightarrow a b}(\sqrt{s}, \cos (\vartheta)) \\
& =\frac{1}{32 \pi s} \frac{p_{\text {out }}}{p_{\text {in }}} \gamma_{i j} \int_{-1}^{1} \mathrm{~d} \cos (\vartheta)|\overline{\mathcal{M}}|^{2}
\end{aligned}
$$

where $\vartheta$ is the final polar angle of one of the final particles in the CM frame, and $\gamma_{i j}=1-\frac{1}{2} \delta_{i j}$ is the symmetry factor.

In this paper we use the short-hand " $\left\{\mu_{q}\right\}$ " instead of " $\mu_{\mathrm{B}}, \mu_{\mathrm{Q}}, \mu_{\mathrm{S}}$ " in function arguments, and the $(+,-,-,-)$ signature for the metric. Greek indices run from 0 to 3 and latin ones run from 1 to 3 . Furthermore, we employ natural units, $\hbar=c=k_{\mathrm{B}}=1$.

\section{A. First-order Chapman-Enskog approximation}

If the perturbations from equilibrium are small, one can expand the single-particle distribution function in orders of the Knudsen number $(\mathrm{Kn})$ :

$$
f_{i, \mathbf{k}}=f_{i, \mathbf{k}}^{(0)}+\epsilon f_{i, \mathbf{k}}^{(1)}+\mathcal{O}\left(\epsilon^{2}\right)
$$

where $\epsilon$ is an assisting parameter for counting the orders of the gradients (or equivalently, the orders of the Knudsen number), which will be send to 1 afterwards. This approximation is known as the Chapman-Enskog expansion to first order (CE) [67]. Neglecting second-order terms leads to the linearized effective Boltzmann equation: 


$$
k_{i}^{\mu} \partial_{\mu} f_{i, \mathbf{k}}^{(0)}+\frac{1}{2} \partial_{\mu}\left(m_{i}^{2}\right) \frac{\partial}{\partial k_{i, \mu}} f_{i, \mathbf{k}}^{(0)}=\sum_{j=1}^{N_{\text {species }}} \mathcal{C}_{i j}^{(1)}\left[f_{i, \mathbf{k}}\right],
$$

with the linearized collision term

$$
\begin{aligned}
\sum_{j=1}^{N_{\text {species }}} \mathcal{C}_{i j}^{(1)}\left[f_{i, \mathbf{k}}\right] \equiv & \frac{1}{2} \sum_{j, a, b=1}^{N_{\text {species }}} \int_{\mathbb{R}^{3}} \mathrm{~d} P_{a} \int_{\mathbb{R}^{3}} \mathrm{~d} P_{b}^{\prime} \int_{\mathbb{R}^{3}} \mathrm{~d} K_{j}^{\prime} \\
& \times W_{k k^{\prime} \rightarrow p p^{\prime}}^{i j \rightarrow a b} f_{i, \mathbf{k}}^{(0)} f_{j, \mathbf{k}^{\prime}}^{(0)} \tilde{f}_{a, \mathbf{p}}^{(0)} \tilde{f}_{b, \mathbf{p}^{\prime}}^{(0)} \\
& \times\left(\frac{f_{a, \mathbf{p}}^{(1)}}{f_{a, \mathbf{p}}^{(0)} \tilde{f}_{a, \mathbf{p}}^{(0)}}+\frac{f_{b, \mathbf{p}^{\prime}}^{(1)}}{f_{b, \mathbf{p}^{\prime}}^{(0)} \tilde{f}_{b, \mathbf{p}^{\prime}}^{(0)}}\right. \\
& \left.-\frac{f_{i, \mathbf{k}}^{(1)}}{f_{i, \mathbf{k}}^{(0)} \tilde{f}_{i, \mathbf{k}}^{(0)}}-\frac{f_{j, \mathbf{k}^{\prime}}^{(1)}}{f_{j, \mathbf{k}^{\prime}}^{(0)} \tilde{f}_{j, \mathbf{k}^{\prime}}^{(0)}}\right)
\end{aligned}
$$

and

$W_{k k^{\prime} \rightarrow p p^{\prime}}^{i j \rightarrow a b} \equiv \frac{(2 \pi)^{4}}{16} \delta^{(4)}\left(k_{i}+k_{j}^{\prime}-p_{a}-p_{b}^{\prime}\right)\left|\overline{\mathcal{M}}_{i j \rightarrow a b}\right|^{2}$

for the inelastic binary transition rate. To linear order the diffusion currents are given via:

$$
V_{q}^{\mu}=\sum_{i=1}^{N_{\text {species }}} q_{i} \int_{\mathbb{R}^{3}} \mathrm{~d} K_{i} k_{i}^{\langle\mu\rangle} f_{i, \mathbf{k}}^{(1)},
$$

and therefore the explicit mass-term in Eq. (13) does not affect the currents due to the antisymmetry of the integrand [17]. Here $q_{i}$ is again the quantum number of type $q \in\{\mathrm{B}, \mathrm{S}, \mathrm{Q}\}$ of the $i$ th particle species.

For further evaluations with the CE method in this study we consider a classical system of on-shell particles, $a_{i}=0 \forall i$, and binary, elastic collisions that are characterized by their angle-integrated, total cross sections, such that the on-shell transition rate for this case reads:

$$
\begin{aligned}
W_{k k^{\prime} \rightarrow p p^{\prime}}^{i j \rightarrow a b} & =\left(\delta_{i a} \delta_{j b}+\delta_{i b} \delta_{j a}\right)(2 \pi)^{6} s \\
& \times \frac{\sigma_{\mathrm{tot}}^{i j \rightarrow i j}}{4 \pi} \delta^{(4)}\left(k_{i}+k_{j}^{\prime}-p_{a}-p_{b}^{\prime}\right) .
\end{aligned}
$$

We remark that, on the level of the CE method, that this effectively renders it an isotropic approach but the integrated cross sections may be taken from theories describing anisotropic interactions.

In the following we will review the CE method presented in previous works $[15,17,40]$, and where it was applied to a variety of hadronic and partonic systems. For these socalled tunes, the thermal quantities (energy density, charge density and pressure), a list of particles and its properties (mass, charge, etc.), and the (total) cross sections of their respected interactions are needed. In this work, we want to apply ("tune") the CE method to the dynamical quasiparticle model (DQPM) and therefore significantly improve our computations for partonic systems from the former publications. In general the DQPM delivers, among others, medium-corrected differential cross sections for anisotropic, inelastic binary processes of partons (see review of the DQPM in Sec. II C). In order to be able to use these in the CE method (as presented here), we evaluate the corresponding total cross section via Eq. (11), and neglect the inelastic channels. It should be noted that these cross sections depend on the collisional energy $\sqrt{s}$, the temperature and the baryon chemical potential, $\sigma_{\text {tot }}^{i j \rightarrow i j} \equiv \sigma_{\text {tot }}^{i j \rightarrow i j}\left(\sqrt{s}, T, \mu_{\mathrm{B}}\right)$. For the convenience of the reader, all underlying assumptions for the CE method will be again summarized in the preface of Sec. III.

Following the steps taken in Refs. $[15,17,40]$, we can express the diffusion coefficient matrix for a classical system under the above given assumptions as

$\kappa_{q q^{\prime}}=\frac{1}{3} \sum_{i=1}^{N_{\text {species }}} q_{i} \sum_{m=0}^{M} \lambda_{m, q^{\prime}}^{(i)} \int_{\mathbb{R}^{3}} \mathrm{~d} K_{i} E_{i, \mathbf{k}}^{m}\left(m_{i}^{2}-E_{i, \mathbf{k}}^{2}\right) f_{i, \mathbf{k}}^{(0)}$,

where the scalars $\lambda_{m, q^{\prime}}^{(i)}$ are solutions of the linearized Boltzmann equation in the form $[15,17,40]$ :

$$
\sum_{m=0}^{M} \sum_{j=1}^{N_{\text {species }}}\left(\mathcal{A}_{n m}^{i} \delta^{i j}+\mathcal{C}_{n m}^{i j}\right) \lambda_{m, q}^{(j)}=b_{q, n}^{i},
$$

with the abbreviations

$$
\begin{aligned}
\mathcal{A}_{n m}^{i} & \equiv \sum_{\ell=1}^{N_{\text {species }}} \int \mathrm{d} K_{i} \mathrm{~d} K_{\ell}^{\prime} \mathrm{d} P_{i} \mathrm{~d} P_{\ell}^{\prime}(2 \pi)^{6} s \frac{\sigma_{\text {tot }}^{i \ell \rightarrow \ell}}{4 \pi} \delta^{(4)}\left(k_{i}+k_{\ell}^{\prime}-p_{i}-p_{\ell}^{\prime}\right) f_{i, \mathbf{k}}^{(0)} f_{\ell, \mathbf{k}^{\prime}}^{(0)} E_{i, \mathbf{k}}^{n-1} k_{i,\langle\alpha\rangle}\left(E_{i, \mathbf{p}}^{m} p_{i}^{\langle\alpha\rangle}-E_{i, \mathbf{k}}^{m} k_{i}^{\langle\alpha\rangle}\right), \\
\mathcal{C}_{n m}^{i j} & \equiv \int \mathrm{d} K_{i} \mathrm{~d} K_{j}^{\prime} \mathrm{d} P_{i} \mathrm{~d} P_{j}^{\prime}(2 \pi)^{6} s \frac{\sigma_{\text {tot }}^{i j \rightarrow i j}}{4 \pi} \delta^{(4)}\left(k_{i}+k_{j}^{\prime}-p_{i}-p_{j}^{\prime}\right) f_{i, \mathbf{k}}^{(0)} f_{j, \mathbf{k}^{\prime}}^{(0)} E_{i, \mathbf{k}}^{n-1} k_{i,\langle\alpha\rangle}\left(E_{j, \mathbf{p}^{\prime}}^{m} p_{j}^{\prime\langle\alpha\rangle}-E_{j, \mathbf{k}^{\prime}}^{m} k_{j}^{\langle\alpha\rangle}\right), \\
b_{q, n}^{i} & \equiv \int_{\mathbb{R}^{3}} \mathrm{~d} K_{i} E_{i, \mathbf{k}}^{n-1}\left(m_{i}^{2}-E_{i, \mathbf{k}}^{2}\right)\left(\frac{E_{i, \mathbf{k}} n_{q}}{\epsilon+P_{0}}-q_{i}\right) f_{i, \mathbf{k}}^{(0)},
\end{aligned}
$$


and where we further impose Landau's definition of the frame [71], which leads to the additional constraint:

$$
\begin{array}{r}
W^{\mu}=\sum_{i=1}^{N_{\text {species }}} \int_{\mathbb{R}^{3}} \mathrm{~d} K_{i} E_{i, \mathbf{k}} k_{i}^{\langle\mu\rangle} f_{i, \mathbf{k}}^{(1)} \stackrel{!}{=} 0 \Rightarrow \\
\sum_{i=1}^{N_{\text {species }}} \sum_{m=0}^{M} \lambda_{m, q}^{(i)} \int_{\mathbb{R}^{3}} \mathrm{~d} K_{i} E_{i, \mathbf{k}}^{m+1}\left(m_{i}^{2}-E_{i, \mathbf{k}}^{2}\right) f_{i, \mathbf{k}}^{(0)} \stackrel{!}{=} 0 .
\end{array}
$$

Above we introduced the truncation order $M$; for the sake of simplicity the order is fixed to $M=1$ which corresponds to the 14-moment approximation [72]. We further define the corresponding conductivities as, $\sigma_{q q^{\prime}} / T=\kappa_{q q^{\prime}} / T^{2}$ and note that for $q=\mathrm{Q}$ or $q^{\prime}=\mathrm{Q}$ they are equivalent to the cross-electric conductivities introduced in Ref. [19]. Especially, $\kappa_{Q Q} / T^{2}=\sigma_{\mathrm{el}} / T$ is the electric conductivity, which was already evaluated in various models [13-15,17-19,28-42].

\section{B. Relaxation time approximation}

Anderson and Witting proposed an approximation to the collision term by defining a governing relaxation time [73]. To first order, we write for each particle species $i$ :

$$
\begin{aligned}
\sum_{j=1}^{N_{\text {species }}} \mathcal{C}_{i j}^{(1)}\left[f_{i, \mathbf{k}}\right] & =-\frac{E_{i, \mathbf{k}}}{\tau_{i}}\left(f_{i, \mathbf{k}}-f_{i, \mathbf{k}}^{(0)}\right) \\
& =-\frac{E_{i, \mathbf{k}}}{\tau_{i}} f_{i, \mathbf{k}}^{(1)}+\mathcal{O}\left(\mathrm{Kn}^{2}\right) .
\end{aligned}
$$

The relaxation time $\tau_{i}$ is related to the scattering rate $\Gamma_{i}\left(\mathbf{k}_{i}, T,\left\{\mu_{q}\right\}\right)$. For binary scattering we may write down the momentum dependent on-shell relaxation time $[60,74,75]$ :

$$
\begin{aligned}
\frac{1}{\tau_{i}\left(\mathbf{k}_{i}, T,\left\{\mu_{q}\right\}\right)} & =\Gamma_{i}\left(\mathbf{k}_{i}, T,\left\{\mu_{q}\right\}\right) \\
& =\sum_{j=1}^{N_{\text {species }}} \frac{1}{2 !} \frac{1}{E_{i, \mathbf{k}}} \sum_{a, b=1}^{N_{\text {species }}} \int_{\mathbb{R}^{3}} \mathrm{~d} K_{j}^{\prime} \mathrm{d} P_{a} \mathrm{~d} P_{b}^{\prime} f_{j, k^{\prime}}^{(0)} \tilde{f}_{a, \mathbf{p}}^{(0)} \tilde{f}_{b, p^{\prime}}^{(0)} W_{k k^{\prime} \rightarrow p p^{\prime}}^{i j \rightarrow a b}
\end{aligned}
$$

From this we can also define the momentum-averaged relaxation time $\tau_{i, 0}$ which may be used instead:

$$
\begin{aligned}
\frac{1}{\tau_{i, 0}\left(T,\left\{\mu_{q}\right\}\right)} & =\Gamma_{i, 0}\left(T,\left\{\mu_{q}\right\}\right) \\
& \equiv \frac{1}{n_{i}} \int_{\mathbb{R}^{3}} \mathrm{~d} K_{i} E_{i, \mathbf{k}} \Gamma_{i}\left(\mathbf{k}_{i}, T,\left\{\mu_{q}\right\}\right) f_{i, \mathbf{k}}^{(0)}
\end{aligned}
$$

with the on-shell particle density of species $i$ :

$$
n_{i}\left(T,\left\{\mu_{q}\right\}\right) \equiv \int_{\mathbb{R}^{3}} \mathrm{~d} K_{i} E_{i, \mathbf{k}} f_{i, \mathbf{k}}^{(0)}
$$

This is also known as the relaxation time approximation (RTA) (cf. [59-62]).

In the classical limit and for the case of elastic, binary processes with geometric cross sections, $\sigma_{\text {tot }}^{i j \rightarrow i j} \equiv \sigma_{\text {tot }}=$ const., using Eq. (17) we can make the usual approximation (see e.g., [17]):

$$
\frac{1}{\tau_{i, 0}\left(T,\left\{\mu_{q}\right\}\right)}=\Gamma_{i, 0}\left(T,\left\{\mu_{q}\right\}\right) \approx \sigma_{\text {tot }} \sum_{j} n_{j}=\sigma_{\text {tot }} n_{\text {tot }} .
$$

Following Ref. [17], the diffusion coefficient matrix in the RTA can be expressed as:

$$
\begin{aligned}
\kappa_{q q^{\prime}}= & \frac{1}{3} \sum_{i=1}^{N_{\text {species }}} q_{i} \int_{\mathbb{R}^{3}} \mathrm{~d} K_{i} \tau_{i, 0}\left(T, \mu_{\mathrm{B}}\right) \frac{1}{E_{i, \mathbf{k}}}\left(m_{i}^{2}-E_{i, \mathbf{k}}^{2}\right) \\
& \times\left(\frac{E_{i, \mathbf{k}} n_{q^{\prime}}}{\epsilon+P_{0}}-q_{i}^{\prime}\right) f_{i, \mathbf{k}}^{(0)} \tilde{f}_{i, \mathbf{k}}^{(0)} .
\end{aligned}
$$

\section{Dynamical quasiparticle model for the quark-gluon plasma}

In the dynamical quasi-particle model (DQPM) [18,43$45,53]$ the properties of the QGP are described in terms of strongly interacting dynamical quasi-particles-quarks and gluons - with medium-adjusted properties. Their properties are constructed such that the equation of state (EoS) from lattice quantum chromodynamics (IQCD) is reproduced above the deconfinement temperature $T_{c}$. These quasiparticles are characterized by broad spectral functions $\rho_{i}$ $(i=q, \bar{q}, g)$, which are assumed to have a Lorentzian form [44-46]. They depend on the parton masses $m_{i}$ and their associated widths $\gamma_{i}$,

$\rho_{i}(\omega, \mathbf{p})=\frac{\gamma_{i}}{\tilde{E}_{i, \mathbf{p}}}\left(\frac{1}{\left(\omega-\tilde{E}_{i, \mathbf{p}}\right)^{2}+\gamma_{i}^{2}}-\frac{1}{\left(\omega+\tilde{E}_{i, \mathbf{p}}\right)^{2}+\gamma_{i}^{2}}\right)$.

Here, we introduced the off-shell energy $\tilde{E}_{i, \mathbf{p}}=\sqrt{\mathbf{p}^{2}+m_{i}^{2}-\gamma_{i}^{2}}$. In the DQPM the effective (squared) coupling constant $g^{2}$ is assumed to depend on temperature $T$ and baryon-chemical potential $\mu_{\mathrm{B}}$ [47,76-78]. At $\mu_{\mathrm{B}}=0$ its temperature-dependence is parametrized via the entropy density $s\left(T, \mu_{\mathrm{B}}=0\right)$ from lattice QCD from Refs. [69,70] in the following way:

$$
g^{2}\left(T, \mu_{\mathrm{B}}=0\right)=d\left(\left(s(T, 0) / s_{\mathrm{SB}}^{\mathrm{QCD}}\right)^{e}-1\right)^{f},
$$


with the Stefan-Boltzmann entropy density $s_{\mathrm{SB}}^{\mathrm{QCD}}=$ $19 / 9 \pi^{2} T^{3}$ and the dimensionless parameters $d=169.934$, $e=-0.178434$ and $f=1.14631$. In order to obtain the coupling constant at finite baryon chemical potential $\mu_{\mathrm{B}}$, we use of the "scaling hypothesis" which assumes that $g^{2}$ is a function of the ratio of the effective temperature $T^{*}=\sqrt{T^{2}+\mu_{B}^{2} /\left(9 \pi^{2}\right)}$ and the $\mu_{\mathrm{B}}$-dependent critical temperature $T_{c}\left(\mu_{\mathrm{B}}\right)$ as [79]:

$$
g^{2}\left(T / T_{c}, \mu_{\mathrm{B}}\right)=g^{2}\left(\frac{T^{*}}{T_{c}\left(\mu_{\mathrm{B}}\right)}, \mu_{\mathrm{B}}=0\right),
$$

with $T_{c}\left(\mu_{\mathrm{B}}\right)=T_{c}\left(\mu_{\mathrm{B}}=0\right) \sqrt{1-\alpha \mu_{\mathrm{B}}^{2}}, T_{c}\left(\mu_{\mathrm{B}}=0\right) \approx 0.158 \mathrm{GeV}$ and $\alpha=0.974 \mathrm{GeV}^{-2}$. The $\left(T, \mu_{\mathrm{B}}\right)$ behavior of the DQPM coupling $g^{2} /(4 \pi)$ is shown in Fig. 9 in Appendix VA. At $\mu_{\mathrm{B}}=0$ one can see a good agreement between the 1QCD evaluation of the QCD running coupling $\alpha_{s}=g^{2} /(4 \pi)$ for $N_{f}=2$ [80] and the DQPM running coupling.

With the coupling $g^{2}$ fixed from $1 \mathrm{QCD}$, one can now specify the dynamical quasiparticle mass (for gluons and quarks) which is assumed to be given by the HTL thermal mass in the asymptotic high-momentum regime by $[46,81]$

$$
\begin{aligned}
m_{g}^{2}\left(T, \mu_{\mathrm{B}}\right) & =\frac{g^{2}\left(T, \mu_{\mathrm{B}}\right)}{6}\left(\left(N_{c}+\frac{N_{f}}{2}\right) T^{2}+\frac{N_{c}}{2} \sum_{q} \frac{\mu_{q}^{2}}{\pi^{2}}\right), \\
m_{q(\bar{q})}^{2}\left(T, \mu_{\mathrm{B}}\right) & =\frac{N_{c}^{2}-1}{8 N_{c}} g^{2}\left(T, \mu_{\mathrm{B}}\right)\left(T^{2}+\frac{\mu_{q}^{2}}{\pi^{2}}\right),
\end{aligned}
$$

where $N_{c}=3$ the number of colors, while $N_{f}=3$ denotes the number of flavors. The strange quark has a larger bare mass which needs to be considered in its dynamical mass. Empirically we find $m_{s}\left(T, \mu_{\mathrm{B}}\right)=m_{u / d}\left(T, \mu_{\mathrm{B}}\right)+\Delta m$ and $\Delta m=30 \mathrm{MeV}$. Furthermore, the quasiparticles in the DQPM have finite widths, which are adopted in the form [22,79]

$$
\gamma_{i}\left(T, \mu_{\mathrm{B}}\right)=\frac{1}{3} C_{i} \frac{g^{2}\left(T, \mu_{\mathrm{B}}\right) T}{8 \pi} \ln \left(\frac{2 c_{m}}{g^{2}\left(T, \mu_{\mathrm{B}}\right)}+1\right),
$$

where we use the QCD color factors for quarks, $C_{q}=C_{F}=\frac{N_{c}^{2}-1}{2 N_{c}}=4 / 3$, and gluons, $C_{g}=C_{A}=N_{c}=3$. Further, we fixed the parameter $c_{m}=14.4$, which is related to the magnetic cutoff. We assume that the width of the strange quark is the same as that for the light $(u, d)$ quarks. The evaluated masses and widths in the DQPM are shown in Fig. 8 in Appendix VA.

With the quasiparticle properties (or propagators) fixed as described above, one can evaluate the entropy density $s\left(T, \mu_{\mathrm{B}}\right)$, the pressure $P_{0}\left(T, \mu_{\mathrm{B}}\right)$ and energy density $\epsilon\left(T, \mu_{\mathrm{B}}\right)$ in a straight forward manner by starting with the entropy density $s^{\text {dqp }}$ and number density $n^{\text {dqp }}$ in the propagator representation from Baym $[82,83]$ and then identifying, $s=s^{\mathrm{dqp}}$ and $n_{B}=n^{\mathrm{dqp}} / 3$ [18]. The isotropic pressure $P_{0}$ can then be obtained by using the Maxwell relation of a grand canonical ensemble:

$$
P_{0}\left(T, \mu_{\mathrm{B}}\right)=P_{0}(T, 0)+\int_{0}^{\mu_{\mathrm{B}}} n_{B}\left(T, \mu_{\mathrm{B}}^{\prime}\right) \mathrm{d} \mu_{\mathrm{B}}^{\prime},
$$

where the pressure for $\mu_{B}=0$ is defined using the entropy density as

$$
P_{0}(T, 0)=P_{0}\left(T_{0}, 0\right)+\int_{T_{0}}^{T} s\left(T^{\prime}, 0\right) \mathrm{d} T^{\prime},
$$

In Eq. (34) the lower bound is chosen between $0.12<$ $T_{0}<0.15 \mathrm{GeV}$ (we use here $T_{0}=0.145 \mathrm{GeV}$ ). $P_{0}\left(T_{0}, 0\right.$ ) is the 1QCD pressure taken from Refs. [69,70]. The energy density $\epsilon$ then follows from the Euler relation

$$
\epsilon=T s-P_{0}+\mu_{\mathrm{B}} n_{\mathrm{B}}
$$

The $\left(T, \mu_{\mathrm{B}}\right)$ behavior of the DQPM scaled pressure $P_{0} / T^{4}$ and scaled energy density $\epsilon / T^{4}$ are shown in Fig. 11 in Appendix VA. In Ref. [49] we found a good agreement between the entropy density $s(T)$, pressure $P_{0}(T)$, energy density $\epsilon(T)$ and interaction measure $I(T)=\epsilon-3 P_{0}$ resulting from the DQPM, and results from $1 \mathrm{QCD}$ obtained by the BMW group $[69,70]$ at $\mu_{\mathrm{B}}=0$ and $\mu_{\mathrm{B}}=400 \mathrm{MeV}$.

From the above parametrizations of the masses, widths and the couplings, cross sections for anisotropic, inelastic binary tree-level QCD interactions with the dressed propagators and dressed couplings, have been evaluated which depend on temperature and baryon-chemical potential $[18,49]$. The corresponding total cross sections are shown in Fig. 9 in Appendix VA, and are used of in the ChapmanEnskog evaluation described in Sec. II A. Further, we provide new results for the complete diffusion coefficient matrix from the DQPM in the RTA by using Eq. (27) and assuming relaxation times from Eq. (23). In the following the results from both approaches are presented.

\section{RESULTS}

We provide first results for the diffusion coefficient matrix for the hot quark-gluon plasma at zero and finite baryon chemical potential $\mu_{\mathrm{B}}$ by applying the ChapmanEnskog method, reviewed in Sec. II A and described in detail in Refs. $[15,17,40]$, to a strongly interacting QGP system described by the DQPM (see Sec. IIC and Ref. [18]). This is meant to be a significant and important improvement to the "simplified" model of a partonic system proposed in Refs. [15,17]. These results-obtained within the Chapman-Enskog method-are further compared to the results for the diffusion coefficient matrix calculated within RTA approach based on the DQPM as well as to various other models. The fact that the linearized Boltzmann equation is solved in the $\mathrm{CE}$ framework implies 

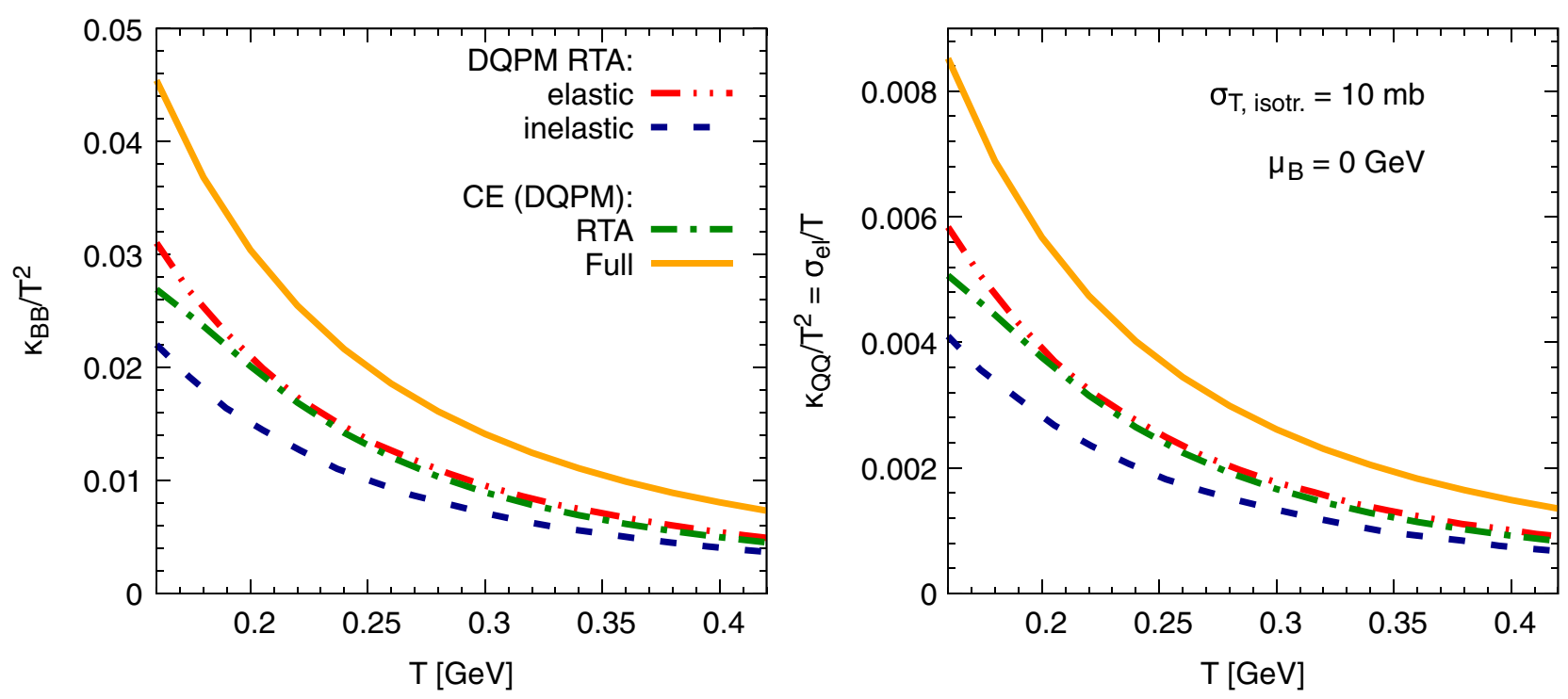

FIG. 1. The scaled baryon diffusion coefficient, $\kappa_{\mathrm{BB}} / T^{2}$ (left), and the scaled electric conductivity, $\sigma_{\mathrm{el}} / T$ (right), for a partonic system with geometric cross sections, $\sigma_{\text {tot }}=10 \mathrm{mb}$ at vanishing baryon-chemical potential as a function of temperature from different approaches. We compare the DQPM RTA $\left(\tau_{i}\left(\mathbf{k}_{i}, T,\left\{\mu_{q}\right\}\right)\right.$ ) results from Eq. (27) (for a system obeying quantum statistics, i.e., $a_{i}= \pm 1$ in Eq. (2) with (blue dashed line) and without (red dashed-double-dotted line) inelastic, flavor-changing channels to the CE (DQPM) results either in RTA $\left(\tau_{0}\left(T,\left\{\mu_{q}\right\}\right)\right.$ ) (green dashed-dotted line) from Eq. (27) [for a system obeying classical statistics, i.e., $a_{i}=0$ in Eq. (2)] or for "full" linearized collision term (orange solid line) from Eq. (18) $\left[a_{i}=0\right.$ in Eq. (2)].

an improvement compared to approaches using the RTA (also see Ref. [17]) in terms of accounting for high moments of the distribution function. However, the proposed Chapman-Enskog method requires a few approximations for the QGP description, which are not in the spirit of the DQPM, in particular:

(1) The system is assumed to obey classical (MaxwellBoltzmann) statistics (i.e., $a_{i}=0$ for all particle species in Eq. (2).

(2) All particles are on-shell, therefore only the polemasses from the DQPM, which depend on temperature and baryon-chemical potential, are assumed but their widths are neglected (for general quasiparticle properties see Appendix VA, the Table I and the Fig. 8).

(3) Inelastic scattering channels are neglected. That implies that flavor-changing processes are not taken into consideration, i.e., $q \bar{q} \rightarrow q^{\prime} \bar{q}^{\prime}$ are not allowed.

(4) All scattering processes are considered to be represented by angle-integrated (total) cross sections via Eq. (11) (i.e., processes are effectively considered to be isotropic). The dependence on $\sqrt{s}$, temperature and baryon-chemical potential is taken into account, $\sigma_{\text {tot }}^{i j \rightarrow i j} \equiv \sigma_{\text {tot }}^{i j \rightarrow i j}\left(\sqrt{s}, T, \mu_{\mathrm{B}}\right)$ (see Appendix VA, the Fig. 10 for an example at $\left.\mu_{\mathrm{B}}=0\right)$.

We note that the CE method can in principle be improved such that approximations (1) and (3) become unnecessary. In Sec. III A we find indication that approximation (3) might have a non-neglectable impact. Such improvements are left for future work. However, the nature of the method makes further improvement of points (2) and (4) difficult and require further detailed study.

The CE evaluations are fed with the DQPM EoS, which is in good agreement with the lattice data (see Sec. II C). Since the net strangeness and net electric densities are not available from the assumed 1QCD results, we compute their values from kinetic theory [see Eq. (6)].

In the following the results from the RTA approach applied to the original DQPM are denoted as "DQPM RTA" while the results from the Chapman-Enskog method applied to the DQPM under assumptions (1)-(4) (as described above) is denoted as "CE (DQPM)." We remind here that for constant cross sections the scaled diffusion coefficients behave as $\kappa_{q q^{\prime}} / T^{2} \sim 1 / T^{2}$, as found in Ref. [17]. Such a decreasing behavior is indeed found in Fig. 1.

\section{A. Model study: A system with geometric cross sections}

In order to evaluate the systematic differences between the DQPM RTA and the CE (DQPM) approaches we perform a here "model study" by assuming a (total) geometric cross section of $\sigma_{\text {tot }}=10 \mathrm{mb}$ for all interactions. For this comparison we consider the same assumptions as described in the preface above, but assume all channels to be characterized by the constant cross section.

In Fig. 1 we show results for the scaled baryon coefficient, $\kappa_{\mathrm{BB}} / T^{2}$ (left plot), and the scaled electric conductivity, $\sigma_{\mathrm{el}} / T$ (right plot) at $\mu_{\mathrm{B}}=0$ in a temperature 
range from $160 \mathrm{MeV}$ to $420 \mathrm{MeV}$. The DQPM RTA calculations are presented for two cases: first, where all binary channels, including the inelastic ones (flavor changing processes $q \bar{q} \rightarrow q^{\prime} \bar{q}^{\prime}$ ), are considered (blue dashed line), and for the case where only the elastic channels are accounted for (red dashed-doubledotted line). For the DQPM RTA results presented in this paper we use Eq. (27), where the momentumdependent relaxation times $\tau_{i}\left(\mathbf{k}_{i}, T,\left\{\mu_{q}\right\}\right)$ from Eq. (23) are used [for a system obeying quantum statistics, i.e., $a_{i}= \pm 1$ in Eq. (2)]. The CE (DQPM) calculations are presented in Fig. 1 also for two cases with only elastic channels: in the first case we evaluate the coefficients in RTA with the help of Eq. (27) [for a system obeying classical statistics, i.e., $a_{i}=0$ in Eq. (2)] under the assumption of the simplistic momentum independent relaxation time $\tau_{0}\left(T,\left\{\mu_{q}\right\}\right)=1 / n_{\text {tot }} \sigma_{\text {tot }}$ (orange solid line), and for the second case (full) we consider the full linearized Boltzmann equation via Eq. (18) (for a system obeying classical statistics, i.e., $a_{i}=0$ in Eq. (2)) (green dashed-dotted line).

This model study shows the influence of the consideration of the linearized Boltzmann equation compared to its relaxation time approximation, and the influence of the inelastic channels compared to its neglect. We find that the consideration of the full linearized collision term effectively reduces the scattering rate of a specific particle species, while in the RTA the scattering rate is overestimated. This is because in the collision term not only the scattering of particles from a specific momentum bin into all other disjoint momentum bins is considered, but also the rescattering into this particular momentum bin is accounted for (gain and loss term). As argued in Ref. [17] such an overestimation of the scattering rate leads to a decrease of the diffusion coefficients from RTA (which are antiproportional to the rate).

Furthermore, we find that the inelastic channels lead to a further decrease of the diffusion coefficients due to the repeated effective increase of the scattering rate as shown in Fig. 1. Comparing the elastic version of the DQPM RTA evaluation with the CE (DQPM) calculation in the RTA limit [Eq. (27)], we find a good agreement of the results at high temperatures. This is expected since the only difference between both calculations-DQPM RTA and CE (DQPM) in the RTA limit-is the consideration of quantum corrections and the more sophisticated (momentum-dependent) relaxation time in DQPM RTA.

\section{B. Diffusion coefficient matrix of the quark-gluon plasma}

In the following we show results for the scaled diffusion coefficient matrix, $\kappa_{q q^{\prime}} / T^{2}$, for the partonic phase from the DQPM (RTA) and CE (DQPM) evaluation under the considerations described in the preface of Sec. III. Additionally we consider two cases: (i) We fix all chemical potentials to zero, $\mu_{q}=0$ $(q=\mathrm{B}, \mathrm{S}, \mathrm{Q})$, and show the temperature dependence of the coefficients.

(ii) We fix the temperature to $T=2 T_{c}\left(\mu_{\mathrm{B}}\right)$, and show their dependence on the baryon chemical potential, $\mu_{\mathrm{B}}$. Here we further set the other chemical potentials to zero, $\mu_{\mathrm{S}}=0$ and $\mu_{\mathrm{Q}}=0$.

For most coefficients we find a rich $\mu_{\mathrm{B}}$-dependence. This dependence originates from the fact that all quarks carry baryon number and thus are sensitive to variations in $\mu_{\mathrm{B}}$. In Ref. [17] the temperature dependence of these transport coefficients was reviewed, and it was found that they roughly scale as $\kappa_{q q^{\prime}} / T^{2} \sim 1 /\left(\sigma_{\mathrm{tot}} T^{2}\right)$. In the case of the DQPM at fixed chemical potential, the cross sections depend on temperature as $\sigma_{\text {tot }} \sim 1 / T^{3}$ or $\sim 1 / T^{4}$ (for the considered temperature range). For moderate temperatures $T_{c}<T<3 T_{c}$ the precise temperature dependence of $\sigma_{\text {tot }}^{i j \rightarrow i j}$ varies for different reactions. It depends on the combinations of $s-, t-, u-$ channels for different partonparton scatterings: for $q-q, q-\bar{q}$ and $q-g$ scatterings $\sigma_{\text {tot }} \sim 1 / T^{3}$, while for the $g-g$ channel the terms $1 / T^{3}$, $1 / T^{4}$ have equivalent contribution to the total cross section $\sigma_{\text {tot }} \sim c_{3} / T^{3}+c_{4} / T^{4}$, where $c_{3}, c_{4}$ depend on $\sqrt{s}, \mu_{\mathrm{B}}$ (see, e.g., Fig. 10 in Appendix VA). The temperature dependence of the cross sections is in accordance with the temperature scaling of the DQPM coupling constant $g^{2}\left(T, \mu_{\mathrm{B}}\right)$ (see, e.g., Fig. 9 in Appendix VA). Averaged over momenta the quark relaxation time decreases with increasing temperature and stays almost constant at high $\mathrm{T}$ and increases with increasing baryon chemical potential (see Fig. 2(b) in Ref. [18]). For temperatures $T>2.5 T_{C}$ this leads to a roughly linear dependence in temperature, $\kappa_{q q^{\prime}} / T^{2} \sim T$, which is demonstrated in the figures below. We remind that the diffusion coefficient matrix is symmetric and therefore we may only show six instead of nine coefficients $[84,85]$. In the following we subdivide the presentation of the conductivities in three sections: electric conductivities $\left(\kappa_{\mathrm{QQ}}, \kappa_{\mathrm{QS}}\right.$ and $\left.\kappa_{\mathrm{QB}}\right)$, strange conductivities $\left(\kappa_{\mathrm{SS}}\right.$ and $\left.\kappa_{\mathrm{SB}}\right)$, and finally the baryon conductivities $\left(\kappa_{\mathrm{BB}}\right)$. Since diffusion coefficients and conductivities are related to each other via temperature, $\kappa_{q q^{\prime}}=\sigma_{q q^{\prime}} T$, we use their denomination interchangeably.

\section{Electric conductivities}

The electric conductivity, $\sigma_{\mathrm{el}} / T$, was evaluated in various models (cf. Refs. [13-15,17-19,28-42,79]). In Fig. 2 we compare the results from DQPM RTA and CE (DQPM) to a variety of models for both, the partonic [13,29,31,32,34,35] and hadronic phase $[15,17,19,30,40,42]$, at $\mu_{q}=0$ in a temperature range between 0 and $3 T_{c}$, where here the deconfinement temperature is $T_{c}=158 \mathrm{MeV}$. The Chapman-Enskog and RTA results for the dimensionless ratio of electric conductivity to temperature $\sigma_{\mathrm{el}} / T$ (later referred to as scaled electric conductivity) for $\mu_{q}=0$ are 

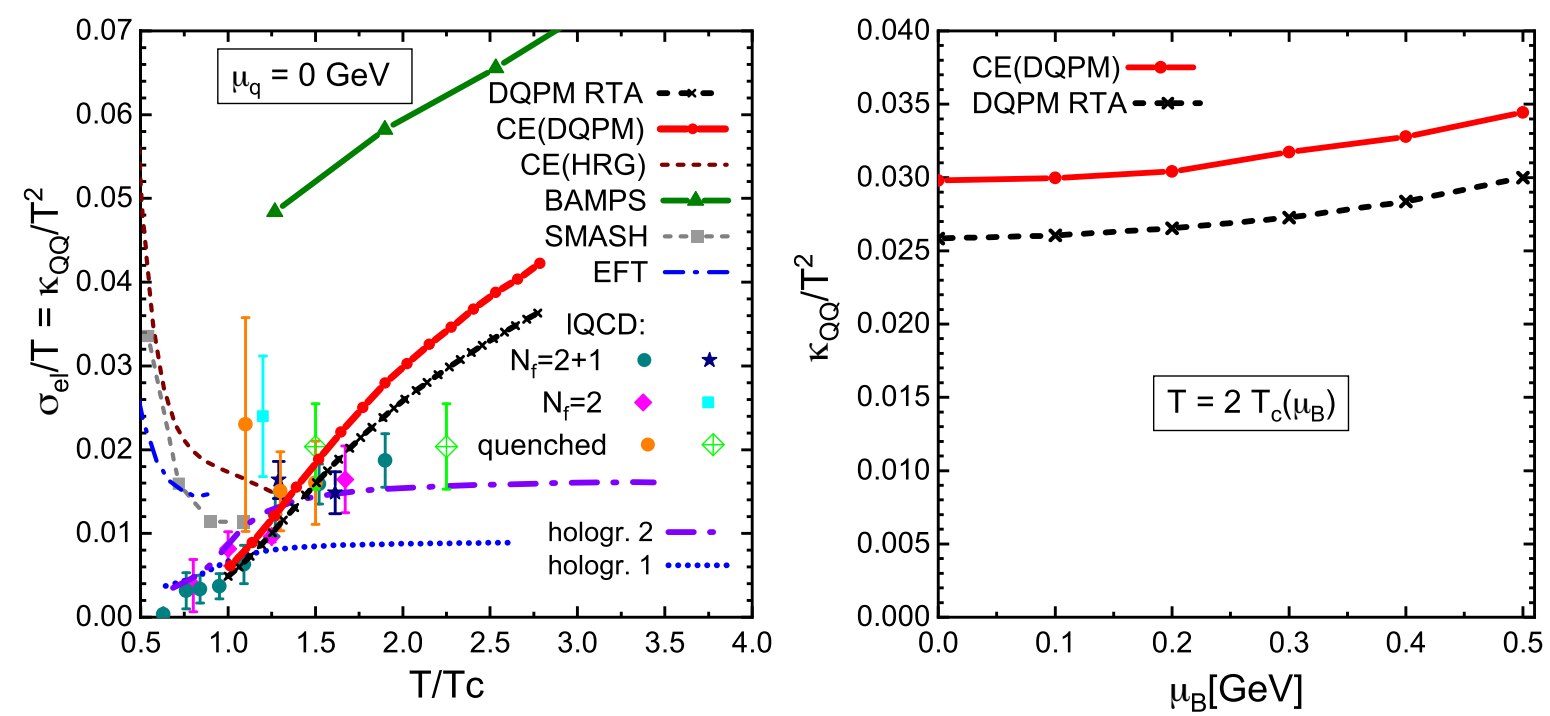

FIG. 2. Left: scaled electric conductivity, $\sigma_{\mathrm{el}} / T=\kappa_{\mathrm{QQ}} / T^{2}$, as a function of the scaled temperature $T / T_{c}$ at vanishing chemical potentials, $\mu_{q}=0$, from various approaches. The results from the CE (DQPM) is shown by the red solid line, and from DQPM RTA-by the black dashed line with crossed points. These are compared to results from the lattice QCD calculations (various shaped points with error bars: quenched: orange circle-shaped points [39], light green rhombus-shaped points [86], $N_{f}=2$ : light cyan circle-shaped points [29], magenta rhombus-shaped points [38], and $N_{f}=2+1$ : dark cyan circle-shaped points [34] and blue stars [87]), the kinetic partonic cascade model BAMPS (dark-green solid line with triangular-shaped points) [35], and from nonconformal holographic models (blue dotted line from Ref. [14] and violet dashed-dotted line from Ref. [31]). For temperatures below $T_{c}=0.158 \mathrm{GeV}$ we show evaluations from hadronic models: SMASH [19,42,88] (grey short-dashed line with squared points), effective field theory (EFT) [30] (blue dashed-dotted line), and CE tuned to a hadron gas [CE (HRG)] from Refs. [15,17,40] (dark-red dashed line). Right: scaled electric conductivity of the QGP at fixed scaled temperature, $T=2 T_{c}\left(\mu_{\mathrm{B}}\right)$, and vanishing $\mu_{\mathrm{Q}}$ and $\mu_{\mathrm{S}}$ are shown for varying baryon chemical potential $\mu_{\mathrm{B}}$ from the DQPM RTA (black dashed line with cross-shaped points) and the CE (DQPM) (red solid line with circle-shaped points) evaluation.

presented in Fig. 2 (left) as solid red and dashed black lines. Results for both methods have a similar increase with temperature, which is mainly a consequence of the temperature dependence of the cross section (as discussed before) and also of the increasing total electric charge density [17].

We find that the results from DQPM RTA and CE (DQPM) are consistent with results from lattice QCD in the vicinity of the crossover region, $1 \leq T / T_{c} \leq 1.5$. We again point out the apparent quadratic dependence on temperature which was shortly motivated in the preface of this section above. Due to our discussion from Sec. III A we suppose that a realistic result for the conductivities may be between the evaluations from DQPM RTA and CE (DQPM).

As follows from Fig. 2, the hadronic models presented there-the hadronic transport model SMASH $[19,42,88]$ (grey short-dashed line with squared points), effective field theory (EFT) [30] (blue dashed-dotted line), and CE tuned to a hadron gas [CE (HRG)] from Refs. [15,17,40] (darkred dashed line) — substantially overestimate the 1QCD data in the vicinity of $T_{c}$ as well as the results from the nonconformal holographic models (blue dotted line from Ref. [13] and violet dashed-dotted line from Ref. [31]). The DQPM RTA results are in a good agreement in the vicinity of phase transition with the previous estimations for
DQPM* from Ref. [79], where nonrelativistic formula for estimation the electric conductivity was used, which results in the linear dependence of the $\sigma_{e l} / T$ on temperature while presented DQPM results show the quadratic dependence on temperature.

Additionally to the electric conductivity, in Fig. 3 we show the cross-electric conductivities, $\sigma_{\mathrm{BQ}}$ and $\sigma_{\mathrm{QS}}$, from the CE (DQPM) and the DQPM RTA calculation together with results achieved within SMASH [19] and the CE (HRG) evaluation from Refs. [15,17] for the same thermal considerations for the hadronic phase. Comparing the results in both phases, we find a significant disagreement for $\sigma_{\mathrm{QB}}$ around the crossover temperature. Further, we find such discrepancies to a smaller extend in the other electric conductivities and in the coefficients to follow. Such disagreement may hint to a difference in the chemical composition of the adjacent phases [19].

Furthermore, as advertised in the preface, in Figs. 2 (right) and 4 we present the sensitivity of the electric conductivities on $\mu_{\mathrm{B}}$ at fixed scaled temperature, $T=2 T_{c}\left(\mu_{\mathrm{B}}\right)$. Compared to the coefficients directly connected to the baryonic sector, we find a rather weak dependence on $\mu_{\mathrm{B}}$ (see discussion of $\kappa_{\mathrm{BB}}$ and $\kappa_{\mathrm{SB}}$ ). Surprisingly also $\sigma_{\mathrm{QB}}$ has such a weak dependence even though it also belongs to the baryonic sector. Further, $\sigma_{\mathrm{QB}}$ is 

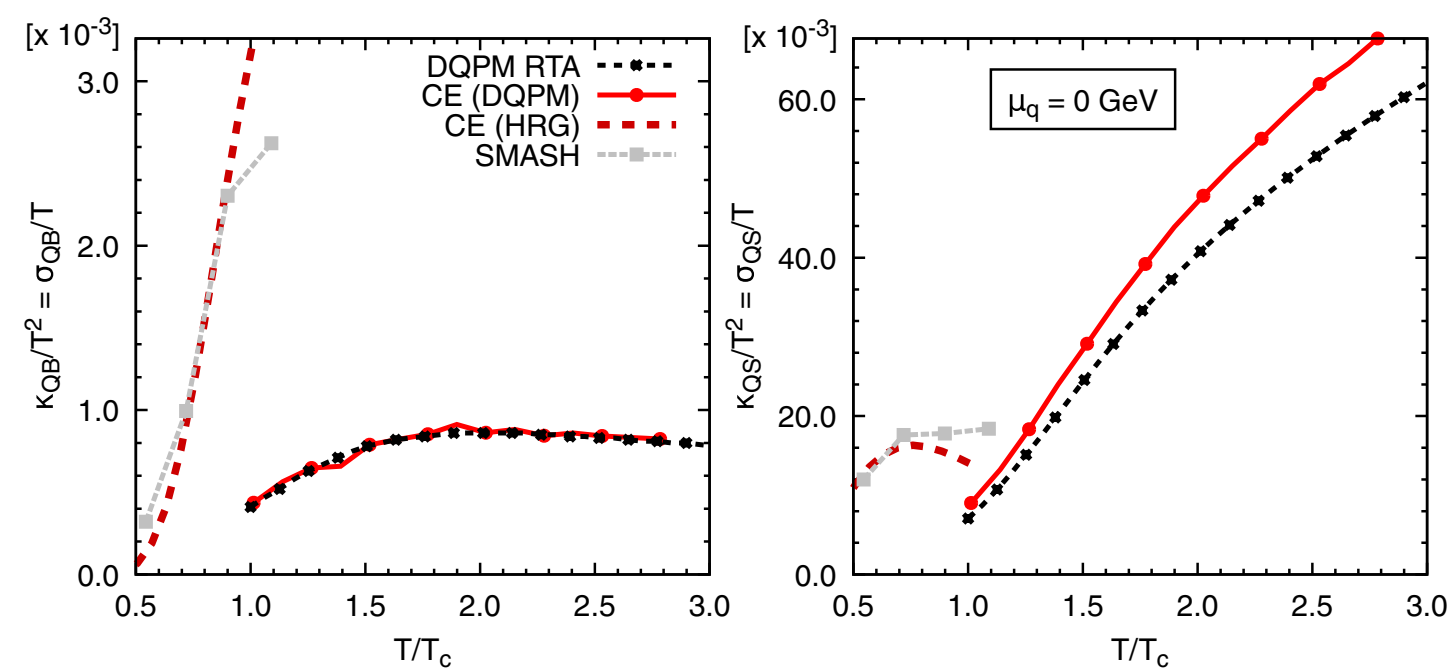

FIG. 3. Scaled cross-electric conductivities, $\sigma_{\mathrm{QB}} / T^{2}$ (left) and $\sigma_{\mathrm{QS}} / T^{2}$ (right), from SMASH [19,88] (grey short-dashed line with square-shaped points), the DQPM RTA (black dashed line with cross-shaped points), and the CE (DQPM) (red solid line with circleshaped points) and CE (HRG) $[15,17]$ (dark-red dashed line) evaluation at vanishing chemical potentials, $\mu_{q}=0$, as a function of scaled temperature in the range from 0.5 to $3 T_{c}$.

very small—it has the smallest magnitude of all conductivities in the diffusion matrix. One can discuss its plausibility with a symmetry argument: the $\sigma_{\mathrm{QB}}$ coefficient relates the generated electric current to the baryonic gradient which generates it (via the corresponding Navier-Stokes term). Assume a QGP with constant geometric cross section as discussed in Sec. III A. Further, assume that all quarks have the same mass. The down- and strange-quark have the same baryon number and electric charge, $\mathrm{B}=+1 / 3$ and $\mathrm{Q}=-1 / 3 e$, while the up-quark has $\mathrm{B}=+1 / 3$ and $\mathrm{Q}=+2 / 3 e$, i.e., the same baryon number but an electric charge which is twice the magnitude but has the opposite sign (refer to Table I). Due to the quarks carrying the same baryon number, a baryon gradient generates a baryon current $V_{\mathrm{B}}^{\mu}$ which is equally composed by a current of up-, down- and strange-quarks $\left(V_{i}^{\mu}\right)$ : $V_{\mathrm{B}}^{\mu}=\sum_{i} \mathrm{~B}_{i} V_{i}^{\mu}$, with $V_{i}^{\mu}=V_{\text {quark }}^{\mu} \forall i$. With this we can estimate the generated electric current:

$$
V_{\mathrm{Q}}^{\mu}=\sum_{i} \mathrm{Q}_{i} V_{i}^{\mu}=V_{\mathrm{quark}}^{\mu}\left(-\frac{1}{3}-\frac{1}{3}+\frac{2}{3}\right)=0
$$

The same argument can be made additionally accounting for the antiquarks. The nonequal mass of the quarks and the

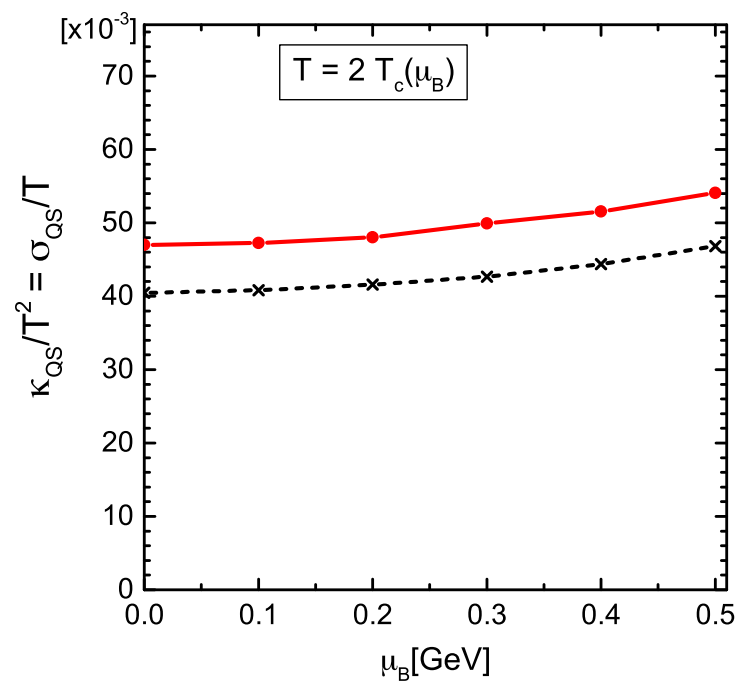

FIG. 4. Scaled cross-electric conductivities, $\sigma_{\mathrm{QB}} / T^{2}$ (left) and $\sigma_{\mathrm{QS}} / T^{2}$ (right), from the DQPM RTA (black dashed line with crossshaped points) and the CE (DQPM) evaluation at fixed scaled temperature, $T=2 T_{c}\left(\mu_{\mathrm{B}}\right)$, shown over baryon chemical potential $\mu_{\mathrm{B}}$ in range 0 to $0.5 \mathrm{GeV}$. Further, the other chemical potentials are fixed to zero, $\mu_{\mathrm{Q}}=0$ and $\mu_{\mathrm{S}}=0$. 

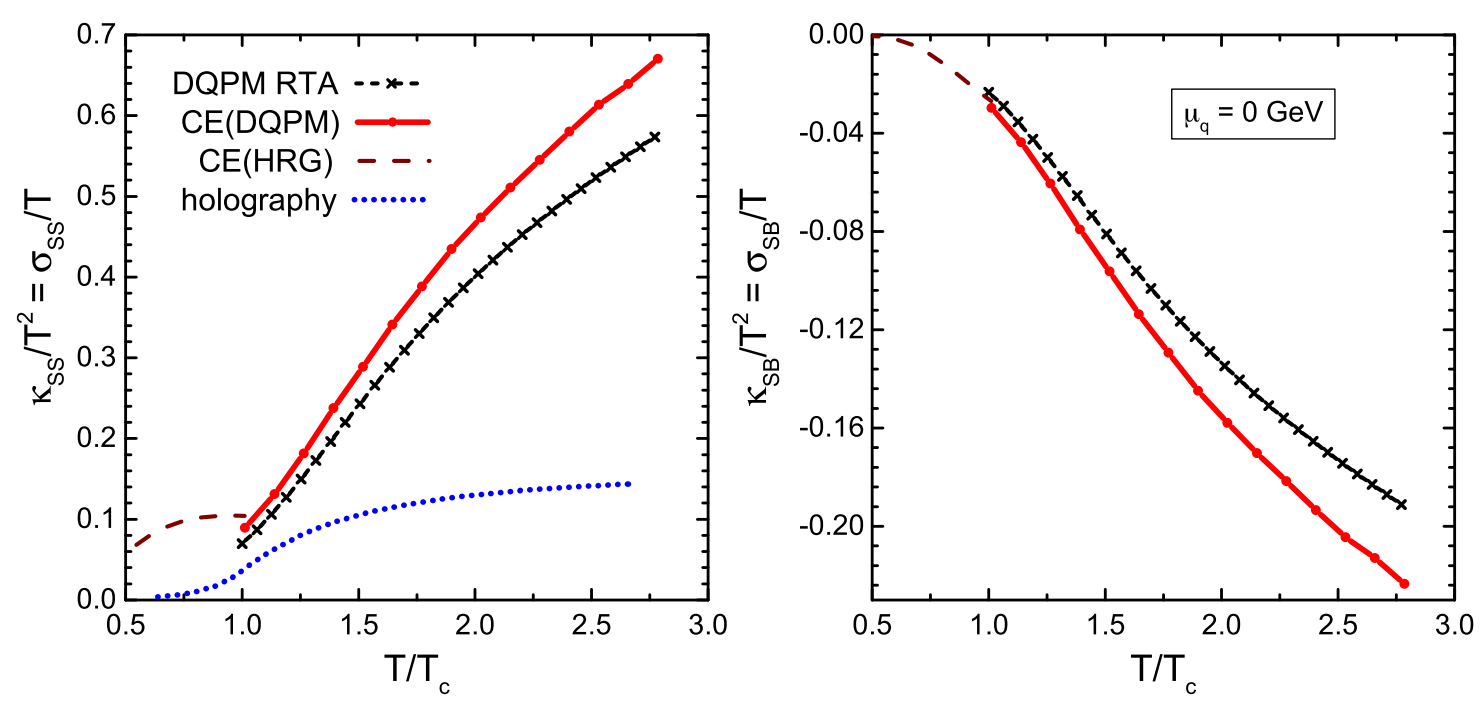

FIG. 5. Scaled strange and strange-baryon diffusion coefficients, $\kappa_{\mathrm{SS}} / T^{2}$ (left) and $\kappa_{\mathrm{SB}} / T^{2}$ (right), as a function of scaled temperature in the range from 0.5 to $3 T_{c}$ at vanishing chemical potentials, $\mu_{q}=0$. We compare results from CE (DQPM) (red solid line with circles), DQPM RTA (black dashed-line with crossed-shaped points), the CE (HRG) [15,17] (dark-red dashed line) and from nonconformal holography [14] (blue dotted line).

varying cross sections lead to a nonvanishing $\sigma_{\mathrm{QB}}$. However, the above estimate illustrate the small magnitude of the respected coefficient.

\section{Strange conductivities}

We continue with the results for the strange sector: $\kappa_{\mathrm{SS}}$ and $\kappa_{\mathrm{SB}}$. The coefficient $\kappa_{\mathrm{SQ}}$, or equivalently $\sigma_{\mathrm{SQ}}=\sigma_{\mathrm{QS}}$, was already discussed above as part of the electric sector. Figure 5 shows the $\kappa_{\mathrm{SS}}$ and $\kappa_{\mathrm{SB}}$ as a function of temperature at vanishing chemical potentials. Further, we show their $\mu_{\mathrm{B}}{ }^{-}$ dependence in Fig. 6 in the range $\mu_{\mathrm{B}}=0$ to $0.5 \mathrm{GeV}$ at fixed scaled temperature, $T=2 T_{c}\left(\mu_{\mathrm{B}}\right)$ and for vanishing electric and strange chemical potential. We compare results from the DQPM RTA and CE (DQPM) computation to results from CE (HRG) in our recent publications $[15,17]$, and to results from nonconformal holography [14].

We find that the baryon-strange diffusion coefficient is negative due to the definition of strangeness carried by the s-quark as has been already advocated in Ref. $[15,17]$. We obtain an almost quadratic dependence in temperature again, and a rather strong dependency on $\mu_{\mathrm{B}}$. However, the results from DQPM RTA for $\kappa_{\mathrm{SB}}$ in Fig. 6 show a slightly different $\mu_{\mathrm{B}}$-behavior than the results from $\mathrm{CE}$
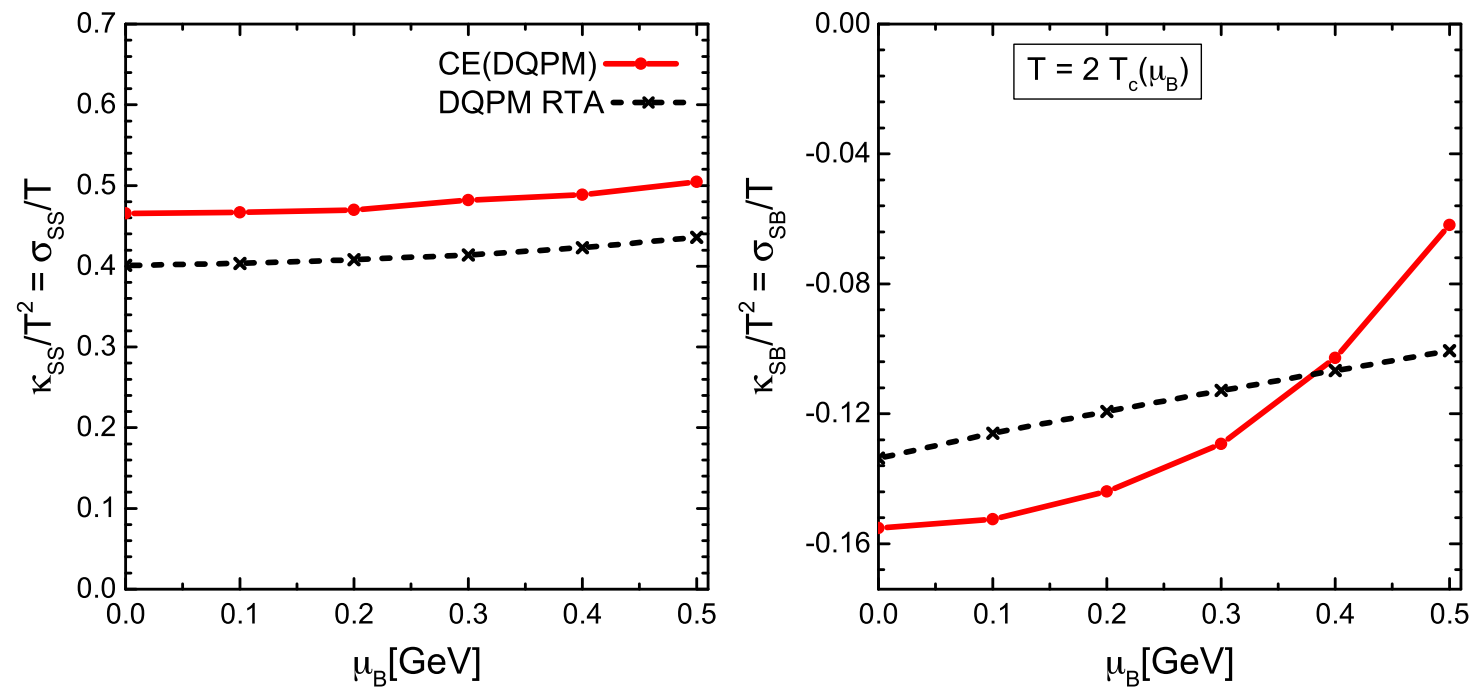

FIG. 6. Scaled strange and strange-baryon diffusion coefficients, $\kappa_{\mathrm{SS}} / T^{2}$ (left) and $\kappa_{\mathrm{SB}} / T^{2}$ (right), from the DQPM RTA (black dashed line with cross-shaped points) and the CE (DQPM) evaluation at fixed scaled temperature, $T=2 T_{c}\left(\mu_{\mathrm{B}}\right)$, shown over baryon chemical potential $\mu_{\mathrm{B}}$ in range 0 to $0.5 \mathrm{GeV}$. Further, the other chemical potentials are fixed to zero, $\mu_{\mathrm{Q}}=0$ and $\mu_{\mathrm{S}}=0$. 

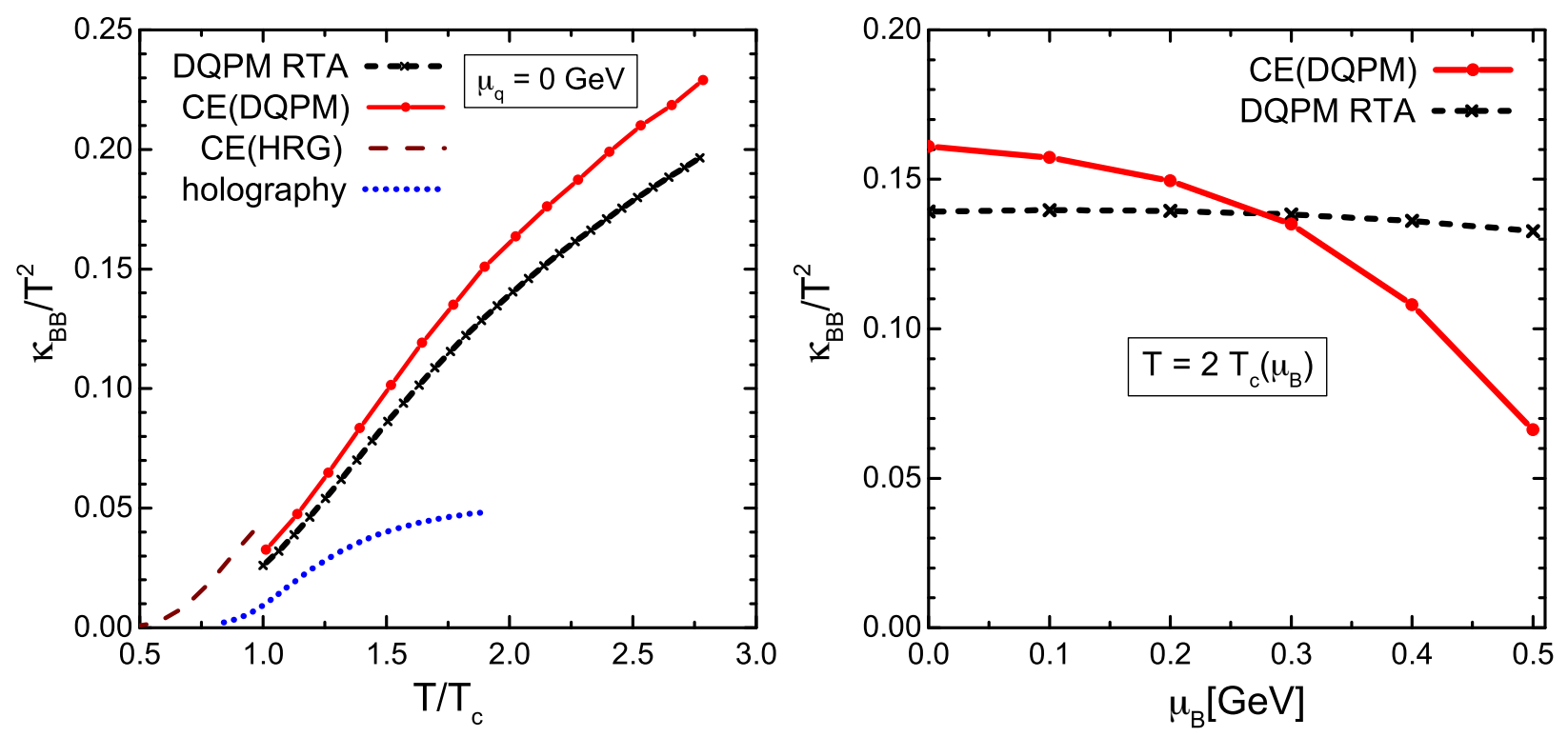

FIG. 7. Scaled baryon diffusion coefficient, $\kappa_{\mathrm{BB}} / T^{2}$, (left) at vanishing chemical potentials, $\mu_{q}=0$, as a function of the scaled temperature from various approaches and (right) at fixed scaled temperature, $T=2 T_{c}\left(\mu_{\mathrm{B}}\right)$, as a function of baryon chemical potential $\mu_{\mathrm{B}}$. The strange and electric potential are fixed to zero: $\mu_{\mathrm{S}}=0$ and $\mu_{\mathrm{Q}}=0$. We show results from the CE evaluation tuned to DQPM (red solid line), as described above, and tuned to a hadron gas from Refs. [15,17] (dark-red dashed line). We again compare to the calculation from DQPM RTA [18] (black dashed line with crosses) and to nonconformal holography [13,14] (blue dotted line) as done for the electric conductivity.

(DQPM) for $\mu_{\mathrm{B}} \geq 0.3 \mathrm{GeV}$. Furthermore, judging from Fig. 5, in the vicinity of the crossover region the results from CE (HRG) for the hadronic phase, and the calculation from the DQPM RTA and CE (DQPM) agree well. As for other diffusion coefficients, the scaled strange diffusion coefficient from holography has a different temperature dependence and smaller values in the vicinity of the crossover phase transition.

\section{Baryon conductivities}

In order to describe the deconfined QCD medium at the nonzero baryon density one should first consider the baryon diffusion coefficient $\kappa_{\mathrm{BB}}$. This diffusion coefficient was already evaluated in various models [12,13,15-18]. Figure 7 (left) shows the temperature dependence of the baryon diffusion coefficient for the quark-gluon plasma estimated with the CE (DQPM) (red solid line) and DQPM RTA approaches (black dashed line with crosses). We also show the results from nonconformal holography $[13,14]$ (blue dotted lines). For temperatures below $T_{c}$ we again refer to the CE (HRG) calculation from Refs. $[15,17]$ (darkred dashed line). The comparison is presented at zero chemical potentials $\mu_{q}=0$. Around $T_{c}$ the results from DQPM RTA, CE (DQPM) and CE (HRG) seem to be rather consistent with each other. Furthermore, we show its dependence on $\mu_{\mathrm{B}}$ at fixed scaled temperature $T=$ $2 T_{c}\left(\mu_{\mathrm{B}}\right)$ in Fig. 7 (right). The DQPM RTA shows a rather weak $\mu_{\mathrm{B}}$ dependence, while $\kappa_{\mathrm{BB}}$ from the CE (DQPM) decreases with $\mu_{\mathrm{B}}$.

\section{CONCLUSION}

In this study we have calculated the complete diffusion coefficient matrix $\kappa_{q q^{\prime}}\left(q, q^{\prime}=\mathrm{B}, \mathrm{S}, \mathrm{Q}\right)$ of the strongly interacting quark-gluon plasma by using the ChapmanEnskog method as well as the relaxation time approximation (RTA) from kinetic theory. We have explored the $T$ and $\mu_{\mathrm{B}}$ dependencies of the diffusion coefficients by considering microscopical properties of quarks and gluons within the dynamical quasiparticle model (DQPM). The DQPM predictions of thermodynamic quantities for finite $\mu_{\mathrm{B}}$ show a good agreement with the available $1 Q C D E o S$ [49]. Moreover, for $\mu_{\mathrm{B}}=0$ the DQPM estimations of the QGP electric conductivity $\left(\sigma_{\mathrm{el}} / T^{2}\right)$ are in a good agreement with the $N_{f}=2+1$ lQCD results and in case of the specific shear and bulk viscosities $(\eta / s, \zeta / s)$ the estimations are remarkably close to the predictions from the gluodynamic IQCD calculations [18].

We find that the electric conductivities $\left(\kappa_{\mathrm{QQ}}, \kappa_{\mathrm{QS}}\right.$ and $\left.\kappa_{\mathrm{QB}}\right)$, strange conductivities $\left(\kappa_{\mathrm{SS}}\right.$ and $\kappa_{\mathrm{SB}}$ ), and finally the baryon conductivity $\left(\kappa_{\mathrm{BB}}\right)$ have a similar temperature dependence in the vicinity of the phase transition while the $\mu_{\mathrm{B}}$ dependence is rather different among the considered diffusion coefficients. In particular, the diffusion coefficients $\kappa_{\mathrm{BB}}$ and $\kappa_{\mathrm{QB}}$ decrease with $\mu_{\mathrm{B}}$, while other coefficients increase. A suppression of baryon diffusion in a sQGP with finite $\mu_{\mathrm{B}}$ has been seen also in the holographic calculations [13].

One of the main endeavors of this paper is to deliver reasonable estimates for the diffusion coefficients of the strongly interacting quark-gluon plasma. Furthermore, we 
compare the RTA evaluations from recent DQPM publications $[18,57,89]$ with the Chapman-Enskog method. We demonstrate that once the cross sections and the (thermal) properties (masses, equation of state, etc.) of a system are known, the CE framework at hand is able to deliver consistent results. We show a good agreement for both methods with the available predictions from the literature for the partonic phase, in particular results for the scaled electric conductivity are remarkably close to the $1 \mathrm{QCD}$ estimates at $\mu_{\mathrm{B}}=0$, as well as with the estimates for the hadronic phase. However, $\kappa_{\mathrm{QB}}$ nondiagonal diffusion coefficient does not coincide well in the vicinity of the phase transition with the estimates for the hadronic phase, which can be interpreted as an indication of a difference in the chemical composition of the adjacent phases. There are several model calculations of diagonal conductivities (mostly $\kappa_{\mathrm{QQ}}$ ) in the literature that are similar to the RTA approach but used numerous restrictive assumptions for the evaluation of the relaxation times or cross sections. Studying the diffusion coefficients of the QGP should have future benefits when considering hydrodynamical description for the time evolution of the deconfined QCD medium.

\section{ACKNOWLEDGMENTS}

The authors acknowledge inspiring discussions with H. van Hees, T. Song and J. M. Torres-Rincon. Also the authors acknowledge support by the Deutsche Forschungsgemeinschaft (DFG, German Research Foundation) through the CRC-TR 211 "Strong-interaction matter under extreme conditions" Project No. 315477589 TRR 211. O. S. and J. A. F. acknowledge support from the Helmholtz Graduate School for Heavy Ion research. Furthermore, we acknowledge support by the Deutsche Forschungsgemeinschaft by the European Unions Horizon 2020 research and innovation program under Grant Agreement No. 824093 (STRONG-2020) and by the COST Action THOR, CA15213. The computational resources have been provided by the LOEWE-Center for Scientific Computing and the "Green Cube" at GSI, Darmstadt.

\section{APPENDIX: PROPERTIES OF PARTONS IN THE DQPM}

TABLE I. Properties of the particle species considered in the quark-gluon plasma in this work. Here, $e$ denotes the elementary electric charge in natural units.

\begin{tabular}{lccccc}
\hline \hline Name & Spin & Degeneracy & Baryon number & Electric charge & Strangeness \\
\hline$g$ & 1 & 16 & 0 & 0 & $+2 / 3 e$ \\
$u$ & $1 / 2$ & 6 & $+1 / 3$ & $-2 / 3 e$ & 0 \\
$\bar{u}$ & $1 / 2$ & 6 & $-1 / 3$ & $-1 / 3 e$ & 0 \\
$d$ & $1 / 2$ & 6 & $+1 / 3$ & $+1 / 3 e$ & 0 \\
$\bar{d}$ & $1 / 2$ & 6 & $-1 / 3$ & $-1 / 3 e$ & 0 \\
$s$ & $1 / 2$ & 6 & $+1 / 3$ & $+1 / 3 e$ & +1 \\
$\bar{s}$ & $1 / 2$ & 6 & $-1 / 3$ & & +1 \\
\hline \hline
\end{tabular}



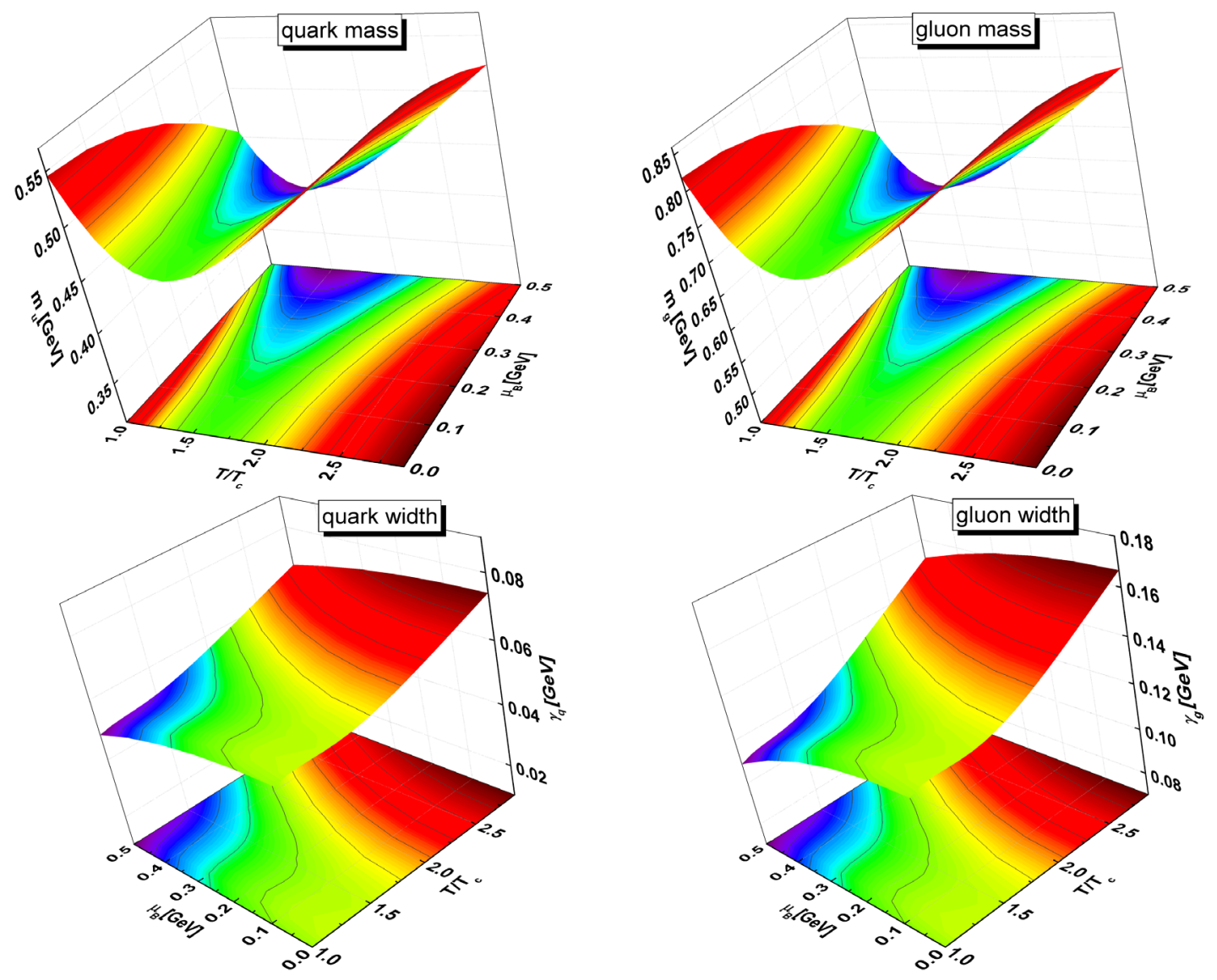

FIG. 8. The effective quark (left) and gluon (right) pole-masses $M$ (upper row) and their widths $\gamma$ (lower row) from the actual DQPM as a function of the temperature $T$ and baryon chemical potential $\mu_{\mathrm{B}}[89]$. The strange quark mass was assumed to be $m_{s}=m_{q}+\Delta m$, with $\Delta m=30 \mathrm{MeV}$, and the width is identical with the widths of the light quarks, $\gamma_{s}=\gamma_{q}$. In the calculation of the diffusion matrix with the Chapman-Enskog method partons were assumed to be on-shell and thus the widths vanish.

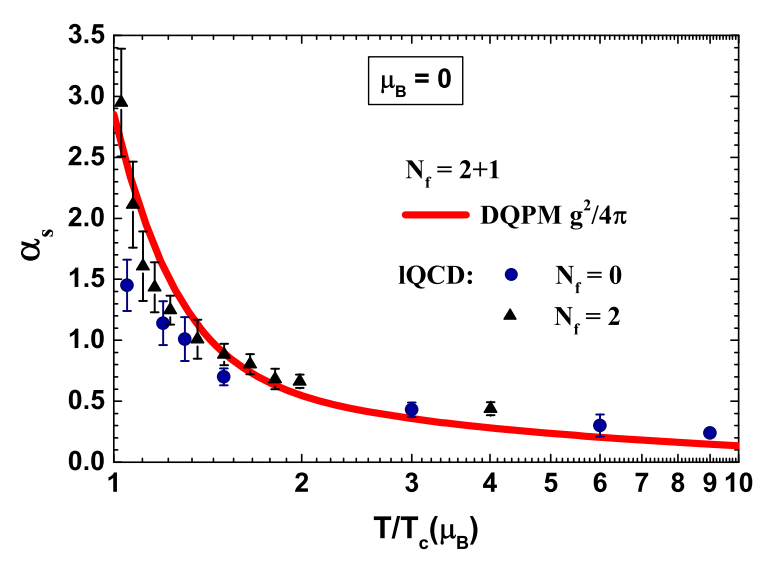

(a)

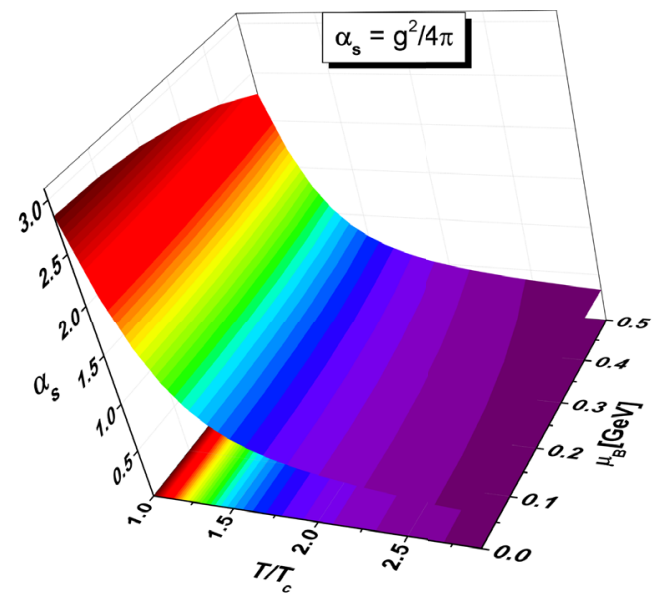

(b)

FIG. 9. The running coupling $\alpha_{s}=g^{2} /(4 \pi)$ from the actual DQPM as a function of the scaled temperature $T / T_{c}$ at $\mu_{\mathrm{B}}=0$ a) and for moderate values of baryon chemical potential $\mu_{\mathrm{B}}<=0.5 \mathrm{GeV}$ (b) [89]. The lattice results for quenched QCD, $N_{f}=0$, (blue circles) are taken from Ref. [48] and for $N_{f}=2$ (black triangles) are taken from Ref. [80]. 


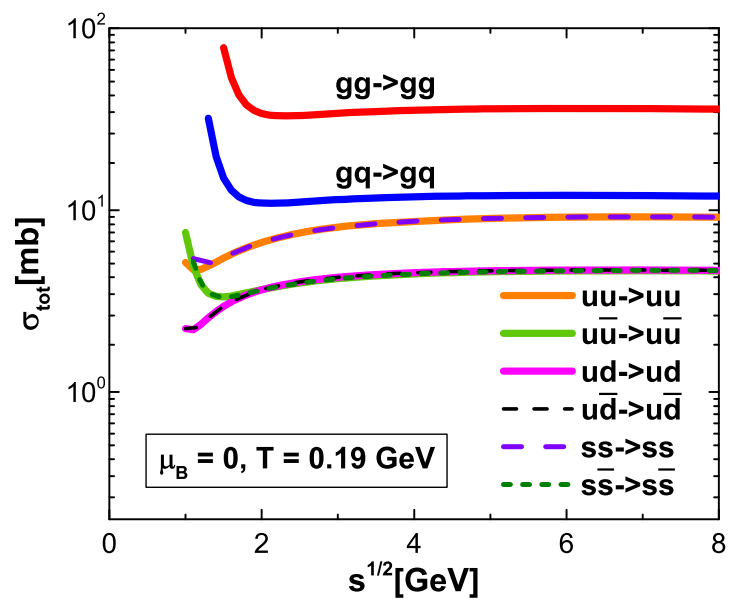

(a)

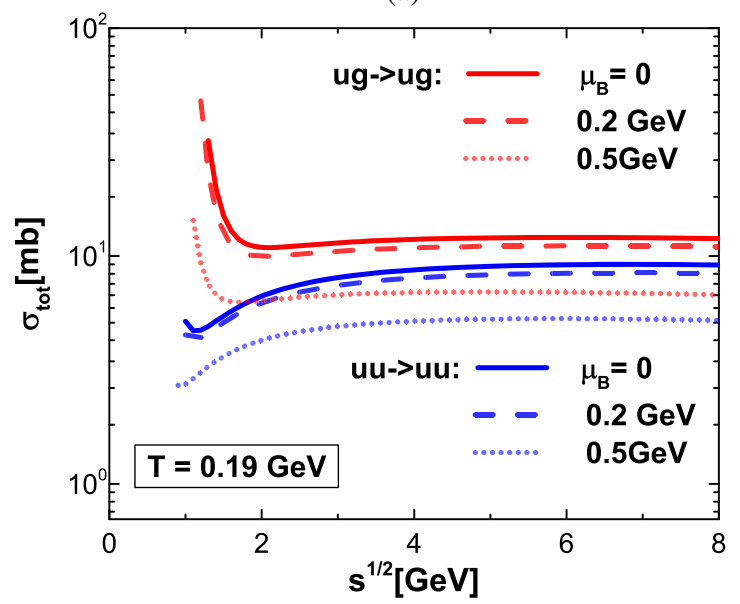

(c)

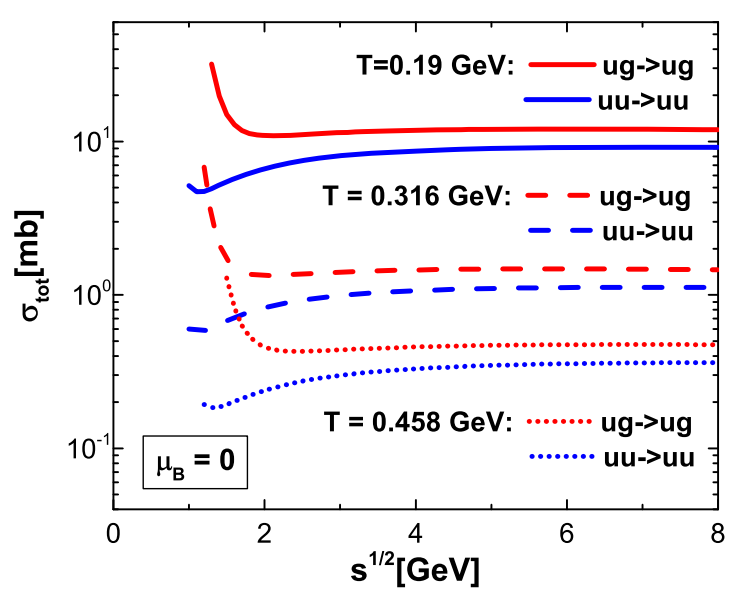

(b)

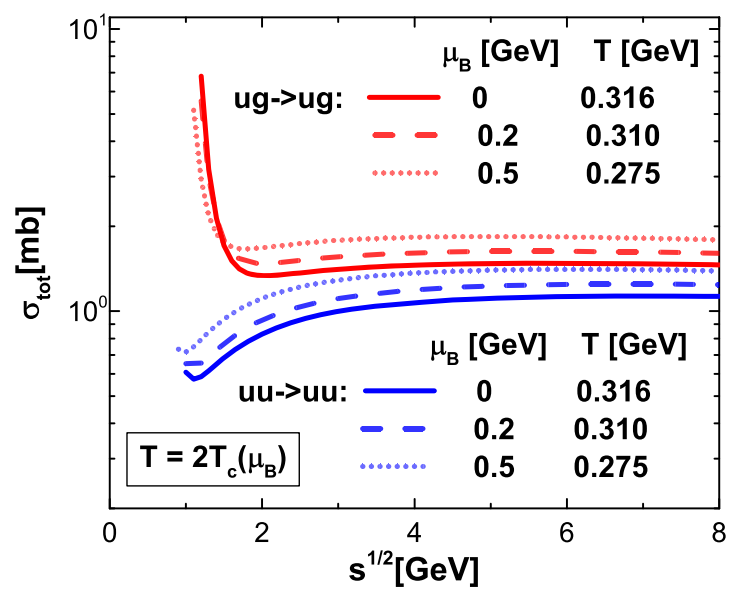

(d)

FIG. 10. DQPM total cross sections between different partons for the on-shell case from Eq. (11) evaluated in the center of mass of the collision system as a function of the collision energy $\sqrt{s}$ for (a) fixed $\mu_{\mathrm{B}}=0, T=0.19 \mathrm{GeV}$ for different elastic channels, (b) for various values of temperature $\mu_{\mathrm{B}}=0, T=0.19,0.316,0.458 \mathrm{GeV}$, (c) various values of baryon chemical potential $\mu_{\mathrm{B}}=0,0.2,0.5 \mathrm{GeV}$ at fixed $T=0.19 \mathrm{GeV}$, and (d) for various values of baryon chemical potential $\mu_{\mathrm{B}}=0,0.2,0.5 \mathrm{GeV}$ for $T=2 T c\left(\mu_{\mathrm{B}}\right)(T=0.316$, $0.310,0.275 \mathrm{GeV})$. For the minimal allowed center-of-momentum energy of the colliding partons only their pole-masses are taken into account. In the Chapman-Enskog evaluation of the diffusion matrix, the inelastic channels were neglected for simplicity.

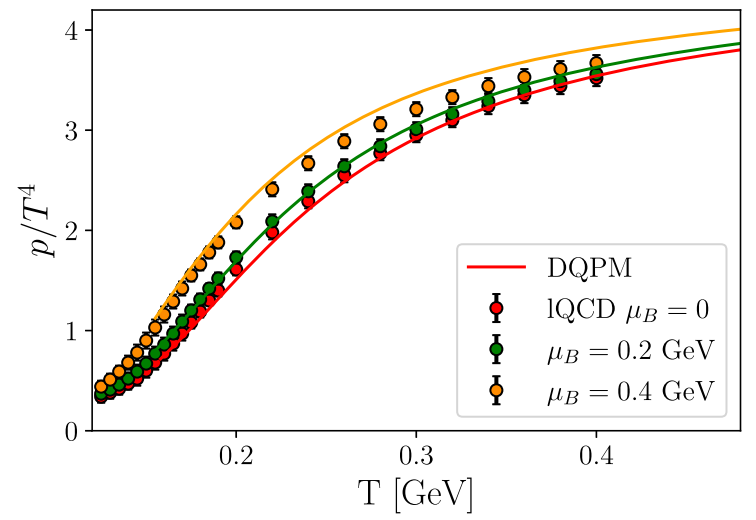

(a)

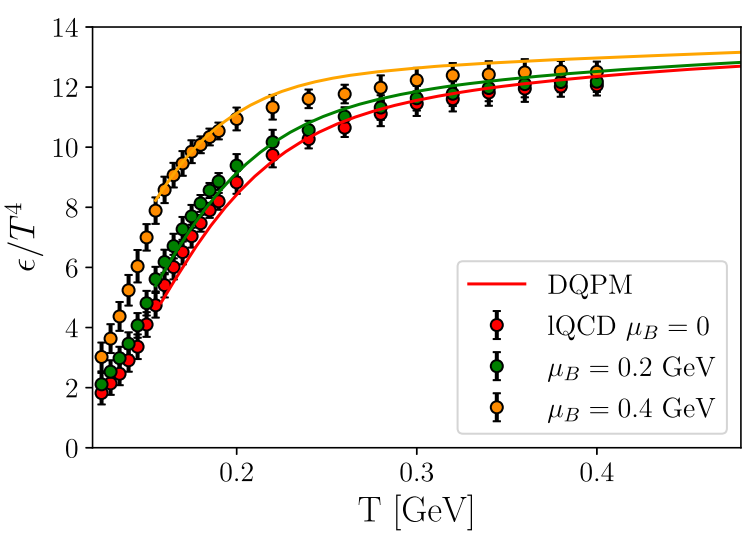

(b)

FIG. 11. Scaled pressure $P_{0} / T^{4}$ (a) and scaled energy density $\epsilon / T^{4}$ (b) from the DQPM (lines) as a function of temperature $T$ at various values of $\mu_{\mathrm{B}}=0,0.2,0.4 \mathrm{GeV}$. The 1QCD results obtained by the BMW group are taken from Refs. [69,70] (circles). 
[1] J.-Y. Ollitrault, Phys. Rev. D 46, 229 (1992).

[2] U. W. Heinz and P. F. Kolb, Nucl. Phys. A702, 269 (2002).

[3] E. Shuryak, Prog. Part. Nucl. Phys. 62, 48 (2009).

[4] E. V. Shuryak, Nucl. Phys. A750, 64 (2005).

[5] M. Gyulassy and L. McLerran, Nucl. Phys. A750, 30 (2005).

[6] P. Romatschke and U. Romatschke, Phys. Rev. Lett. 99, 172301 (2007).

[7] M. Cheng et al., Phys. Rev. D 77, 014511 (2008).

[8] Y. Aoki, S. Borsanyi, S. Durr, Z. Fodor, S. D. Katz, S. Krieg, and K. K. Szabo, J. High Energy Phys. 06 (2009) 088.

[9] G. S. Denicol, C. Gale, S. Jeon, A. Monnai, B. Schenke, and C. Shen, Phys. Rev. C 98, 034916 (2018).

[10] M. Li and C. Shen, Phys. Rev. C 98, 064908 (2018).

[11] L. Du and U. Heinz, Comput. Phys. Commun. 251, 107090 (2020).

[12] P. B. Arnold, G. D. Moore, and L. G. Yaffe, J. High Energy Phys. 05 (2003) 051.

[13] R. Rougemont, J. Noronha, and J. Noronha-Hostler, Phys. Rev. Lett. 115, 202301 (2015).

[14] R. Rougemont, R. Critelli, J. Noronha-Hostler, J. Noronha, and C. Ratti, Phys. Rev. D 96, 014032 (2017).

[15] M. Greif, J. A. Fotakis, G. S. Denicol, and C. Greiner, Phys. Rev. Lett. 120, 242301 (2018).

[16] J. Ghiglieri, G. D. Moore, and D. Teaney, J. High Energy Phys. 03 (2018) 179.

[17] J. A. Fotakis, M. Greif, C. Greiner, G. S. Denicol, and H. Niemi, Phys. Rev. D 101, 076007 (2020).

[18] O. Soloveva, P. Moreau, and E. Bratkovskaya, Phys. Rev. C 101, 045203 (2020).

[19] J.-B. Rose, M. Greif, J. Hammelmann, J. A. Fotakis, G. S. Denicol, H. Elfner, and C. Greiner, Phys. Rev. D 101, 114028 (2020).

[20] S. Turbide, R. Rapp, and C. Gale, Phys. Rev. C 69, 014903 (2004).

[21] Y. Akamatsu, H. Hamagaki, T. Hatsuda, and T. Hirano, J. Phys. G 38, 124184 (2011).

[22] O. Linnyk, E. L. Bratkovskaya, and W. Cassing, Prog. Part. Nucl. Phys. 87, 50 (2016).

[23] Y. Yin, Phys. Rev. C 90, 044903 (2014).

[24] K. Tuchin, Phys. Rev. C 88, 024911 (2013).

[25] G. Inghirami, M. Mace, Y. Hirono, L. Del Zanna, D. E. Kharzeev, and M. Bleicher, Eur. Phys. J. C 80, 293 (2020).

[26] G. S. Denicol, E. Molnár, H. Niemi, and D. H. Rischke, Phys. Rev. D 99, 056017 (2019).

[27] L. Oliva, Eur. Phys. J. A 56, 255 (2020).

[28] P. B. Arnold, G. D. Moore, and L. G. Yaffe, J. High Energy Phys. 11 (2000) 001.

[29] B. B. Brandt, A. Francis, H. B. Meyer, and H. Wittig, J. High Energy Phys. 03 (2013) 100.

[30] J. M. Torres-Rincon, Hadronic transport coefficients from effective field theories, Ph.D. thesis, Universidad Complutense de Madrid, 2012.

[31] S. I. Finazzo and J. Noronha, Phys. Rev. D 89, 106008 (2014).

[32] A. Amato, G. Aarts, C. Allton, P. Giudice, S. Hands, and J.-I. Skullerud, Phys. Rev. Lett. 111, 172001 (2013).

[33] R. Marty, E. Bratkovskaya, W. Cassing, J. Aichelin, and H. Berrehrah, Phys. Rev. C 88, 045204 (2013).
[34] G. Aarts, C. Allton, A. Amato, P. Giudice, S. Hands, and J.-I. Skullerud, J. High Energy Phys. 02 (2015) 186.

[35] M. Greif, I. Bouras, C. Greiner, and Z. Xu, Phys. Rev. D 90, 094014 (2014).

[36] A. Puglisi, S. Plumari, and V. Greco, Phys. Rev. D 90, 114009 (2014).

[37] A. Puglisi, S. Plumari, and V. Greco, Phys. Lett. B 751, 326 (2015).

[38] B. B. Brandt, A. Francis, B. Jaeger, and H. B. Meyer, Phys. Rev. D 93, 054510 (2016).

[39] H.-T. Ding, O. Kaczmarek, and F. Meyer, Phys. Rev. D 94, 034504 (2016).

[40] M. Greif, C. Greiner, and G. S. Denicol, Phys. Rev. D 93, 096012 (2016); 96, 059902(E) (2017).

[41] L. Thakur, P. K. Srivastava, G. P. Kadam, M. George, and H. Mishra, Phys. Rev. D 95, 096009 (2017).

[42] J. Hammelmann, J. M. Torres-Rincon, J.-B. Rose, M. Greif, and H. Elfner, Phys. Rev. D 99, 076015 (2019).

[43] A. Peshier and W. Cassing, Phys. Rev. Lett. 94, 172301 (2005).

[44] W. Cassing, Nucl. Phys. A795, 70 (2007).

[45] W. Cassing, Nucl. Phys. A791, 365 (2007).

[46] O. Linnyk, E. Bratkovskaya, and W. Cassing, Prog. Part. Nucl. Phys. 87, 50 (2016).

[47] H. Berrehrah, E. Bratkovskaya, T. Steinert, and W. Cassing, Int. J. Mod. Phys. E 25, 1642003 (2016).

[48] O. Kaczmarek, F. Karsch, F. Zantow, and P. Petreczky, Phys. Rev. D 70, 074505 (2004); 72, 059903(E) (2005).

[49] P. Moreau, O. Soloveva, L. Oliva, T. Song, W. Cassing, and E. Bratkovskaya, Phys. Rev. C 100, 014911 (2019).

[50] B. Vanderheyden and G. Baym, J. Stat. Phys. 93, 843 (1998).

[51] L. Kadanoff and G. Baym, Quantum Statistical Mechanics (Benjamin, New York, 1962), p. 203.

[52] W. Cassing and E. Bratkovskaya, Phys. Rev. C 78, 034919 (2008).

[53] W. Cassing, Eur. Phys. J. Special Topics 168, 3 (2009).

[54] W. Cassing and E. Bratkovskaya, Nucl. Phys. A831, 215 (2009).

[55] E. Bratkovskaya, W. Cassing, V. Konchakovski, and O. Linnyk, Nucl. Phys. A856, 162 (2011).

[56] V. Ozvenchuk, O. Linnyk, M. Gorenstein, E. Bratkovskaya, and W. Cassing, Phys. Rev. C 87, 024901 (2013).

[57] O. Soloveva, P. Moreau, L. Oliva, V. Voronyuk, V. Kireyeu, T. Song, and E. Bratkovskaya, Particles 3, 178 (2020).

[58] P. Moreau, O. Soloveva, I. Grishmanovskii, V. Voronyuk, L. Oliva, T. Song, V. Kireyeu, G. Coci, and E. Bratkovskaya, Astron. Nachr. 1 (2021).

[59] A. Hosoya and K. Kajantie, Nucl. Phys. B250, 666 (1985).

[60] P. Chakraborty and J. Kapusta, Phys. Rev. C 83, 014906 (2011).

[61] M. Albright and J. Kapusta, Phys. Rev. C 93, 014903 (2016).

[62] S. Gavin, Nucl. Phys. A435, 826 (1985).

[63] R. Kubo, J. Phys. Soc. Jpn. 12, 570 (1957).

[64] G. Aarts and J. M. Martinez Resco, J. High Energy Phys. 04 (2002) 053.

[65] D. Fernandez-Fraile and A. Gomez Nicola, Phys. Rev. D 73, 045025 (2006). 
[66] R. Lang, N. Kaiser, and W. Weise, Eur. Phys. J. A 51, 127 (2015).

[67] S. Chapman and T. G. Cowling, The Mathematical Theory of Non-Uniform Gases: An Account of the Kinetic Theory of Viscosity, Thermal Conduction and Diffusion in Gases (Cambridge University Press, Cambridge, England, 1970).

[68] P. Romatschke, Phys. Rev. D 85, 065012 (2012).

[69] S. Borsanyi, G. Endrodi, Z. Fodor, S. D. Katz, S. Krieg, C. Ratti, and K. K. Szabo, J. High Energy Phys. 08 (2012) 053.

[70] S. Borsanyi, Z. Fodor, C. Hoelbling, S. D. Katz, S. Krieg, and K. K. Szabo, Phys. Lett. B 730, 99 (2014).

[71] L. D. Landau and E. M. Lifschitz, Course of Theoretical Physics. Vol. 6: Fluid Mechanics (Pergamon Press, London, 1959).

[72] G. S. Denicol, H. Niemi, E. Molnár, and D. H. Rischke, Phys. Rev. D 85, 114047 (2012).

[73] J. Anderson and H. Witting, Physica (Amsterdam) 74, 466 (1974).

[74] E. Braaten and M. H. Thoma, Phys. Rev. D 44, 1298 (1991).

[75] M. H. Thoma, Phys. Rev. D 49, 451 (1994).

[76] H. Berrehrah, E. Bratkovskaya, W. Cassing, P. Gossiaux, J. Aichelin, and M. Bleicher, Phys. Rev. C 89, 054901 (2014).

[77] H. Berrehrah, E. Bratkovskaya, W. Cassing, and R. Marty, J. Phys. Conf. Ser. 612, 012050 (2015).
[78] H. Berrehrah, E. Bratkovskaya, W. Cassing, P. B. Gossiaux, and J. Aichelin, Phys. Rev. C 91, 054902 (2015).

[79] H. Berrehrah, E. Bratkovskaya, T. Steinert, and W. Cassing, Int. J. Mod. Phys. E 25, 1642003 (2016).

[80] O. Kaczmarek and F. Zantow, Phys. Rev. D 71, 114510 (2005).

[81] M. L. Bellac, Thermal Field Theory, Cambridge Monographs on Mathematical Physics (Cambridge University Press, Cambridge, England, 2011).

[82] B. Vanderheyden and G. Baym, J. Stat. Phys. 93, 843 (1998);

[83] J. P. Blaizot, E. Iancu, and A. Rebhan, Phys. Rev. D 63, 065003 (2001).

[84] L. Onsager, Phys. Rev. 37, 405 (1931).

[85] L. Onsager, Phys. Rev. 38, 2265 (1931).

[86] G. Aarts, C. Allton, J. Foley, S. Hands, and S. Kim, Phys. Rev. Lett. 99, 022002 (2007).

[87] N. Astrakhantsev, V. V. Braguta, M. D’Elia, A. Y. Kotov, A. A. Nikolaev, and F. Sanfilippo, Phys. Rev. D 102, 054516 (2020).

[88] J. Weil et al., Phys. Rev. C 94, 054905 (2016).

[89] O. Soloveva, P. Moreau, L. Oliva, T. Song, Cassing, and E. Bratkovskaya, J. Phys. Conf. Ser. 1602, 012012 (2020). 